\title{
Synergistic Network Pharmacology for Traditional Chinese Medicine Liangxue Tongyu Formula in Acute Intracerebral Hemorrhagic Stroke
}

\author{
Yang Chen, ${ }^{1,2}$ Ju Dong, ${ }^{1}$ Dongqing Yang, ${ }^{1}$ Qin Qian, ${ }^{1}$ Pengcheng Wang, ${ }^{1}$ Xiaojuan Yang, \\ Wei Li, ${ }^{1}$ Guochun Li ${ }^{1},{ }^{1}$ Xu Shen $\mathbb{D}^{2},{ }^{2}$ and Fushun Wang ${ }^{3}{ }^{3}$ \\ ${ }^{1}$ Department of Public Health of Nanjing University of Chinese Medicine, 210023 Nanjing, Jiangsu Province, China \\ ${ }^{2}$ State Key Laboratory Cultivation Base for TCM Quality and Efficacy, Nanjing University of Chinese Medicine, 210023 Nanjing, \\ Jiangsu Province, China \\ ${ }^{3}$ Institute of Brain and Psychology, Sichuan Normal University, 610060 Chengdu, Sichuan Province, China
}

Correspondence should be addressed to Guochun Li; liguochuncn@126.com, Xu Shen; xshen@njucm.edu.cn, and Fushun Wang; 13814541138@163.com

Received 12 May 2020; Revised 22 June 2020; Accepted 5 February 2021; Published 27 February 2021

Academic Editor: J. Michael Wyss

Copyright (c) 2021 Yang Chen et al. This is an open access article distributed under the Creative Commons Attribution License, which permits unrestricted use, distribution, and reproduction in any medium, provided the original work is properly cited.

Background. Nowadays, acute intracerebral hemorrhage stroke (AICH) still causes higher mortality. Liangxue Tongyu Formula (LXTYF), originating from a traditional Chinese medicine (TCM) prescription, is widely used as auxiliary treatment for AICH. Objective. To dig into the multicomponent, multitarget, and multipathway mechanism of LXTYF on treating AICH via network pharmacology and RNA-seq. Methods. Network pharmacology analysis was used by ingredient collection, target exploration and prediction, network construction, and Gene Ontology (GO) and KEGG analysis, with the Cytoscape software and ClusterProfiler package in R. The RNA-seq data of the AICH-rats were analyzed for differential expression and functional enrichments. Herb-Compound-Target-Pathway (H-C-T-P) network was shown to clarify the mechanism of LXTYF for AICH. Results. 76 active ingredients (quercetin, Alanine, kaempferol, etc.) of LXTYF and 376 putative targets to alleviate AICH (PTGS2, PTGS1, ESR1, etc.) were successfully identified. The protein-protein interaction (PPI) network indicated the important role of STAT3. The functional enrichment of GO and KEGG pathway showed that LXTYF is most likely to influence MAPK and PI3K-Akt signaling pathways for AICH treatment. From the RNA-seq of AICH-rats, 583 differential mRNAs were identified and 14 of them were consistent with the putative targets of LXTYF for AICH treatment. The KEGG pathway enrichment also implied that the MAPK signaling pathway was the most correlated one among all the related signaling pathways. Many important targets with expression changes of LXTYF for AICH treatment and their related pathways are great markers of antioxidation, anti-inflammatory, antiapoptosis, and lowering blood pressure, which indicated that LXTYF may play mutiroles in the mechanisms for AICH treatment. Conclusion. The LXTYF attenuates AICH partially by antioxidation, antiinflammatory, and antiapoptosis and lowers blood pressure roles through regulating the targets involved MAPK, calcium, apoptosis, and TNF signaling pathway, which provide notable clues for further experimental validation.

\section{Introduction}

Nowadays, stroke is still a major threat to people's health. Statistics from the World Health Organization show that 15 million people suffer the disease worldwide each year [1]. Less frequently than ischemic strokes, intracerebral hemorrhage (ICH) makes up $10 \%-20 \%$ of all strokes. But a higher fatality rate is caused in the acute phase of intracerebral hemorrhage (AICH), up to $40 \%-54 \%[2,3]$. ICH leads to stroke damage when a troubled brain blood vessel bursts, blood leaks, and the brain is loss of oxygen and blood. Deep brain and nerve damage is made due to the dead blood clot cells and released toxins. High blood pressure is the most common cause of ICH [4]. Various forms 
of surgical decompression are in widespread use to treat this kind of stroke, but the effect remains controversial. Unfortunately, there is still no proven (in phase 3 trials) beneficial medical treatment for $\operatorname{AICH}[5,6]$.

In the last two thousand years, traditional Chinese medicines (TCMs) have been applied in treating various kinds of diseases in China, including stroke $[7,8]$. Compared with Western medical treatment for AICH, TCMs have fewer adverse effects and holistic and systematic influence on more pharmacological activities and efficacies. Currently, an important challenge is to discover proven therapeutic medication for AICH. Buyang Huanwu Decoction, Fufang Danshen Injection, Xueshuantong Injection, and Liangxue Tongyu Formula are of the most common use for $\mathrm{ICH}$ herbal patent injections [9]. Among them, Liangxue Tongyu Formula (LXTYF) is a good prescription beneficial for ameliorating $\mathrm{AICH}$, created by Chinese Medical Master Zhongying Zhou, who has 70 years of medical experience in China. It comprises six botanical medicinal materials like Rheum palmatum L. (Dahuang, DaH), Rehmannia glutinosa Libosch. (Dihuang, DiH), Panax notoginseng (Burk.) F.H.Chen (Sanqi, SQ), Paeonia lactiflora Pall. (Chishao, CS), Paeonia suffruticosa Andr (Mudanpi, MDP), and Acori Tatarinowii Schott (Shichangpu, SCP) and two animal medicines like Bubali Cornu (Shuiniujiao, SNJ), Pheretima aspergillum (E. Perrier) (Dilong, DL). This formula was adapted from Xijiao Dihuang Decoction, an ancient TCM classical prescriptions reserved in one of the classic works of ancient Chinese medicine called Essential Recipes for Emergent Use Worth A Thousand Gold, and only replaced Rhinoceri Asiatici Cornu (rhinoceros horn) with SNJ for the similar medicinal effects and compliance with animal protection and regulations [10]. According to previous research, LXTYF may improve symptoms of AICH, attenuate nerve injury, and remove blood stasis of patients, as well as ameliorate brain edemas in AICH rats [11, 12]. For Ma's $96 \mathrm{AICH}$ patients and Guo's $168 \mathrm{AICH}$ patients in treatment group, LXTYF combined with Western medicine treatment was reported to have a better therapeutic effect than Western medication only $[12,13]$. It attenuated symptoms of $\mathrm{AICH}$, promoted the absorption of intracerebral hematoma, improved the recovery of consciousness, and reduced the nerve damage of patients, thus improving the prognosis and reduced the disability rate $[12,13]$. Li's previous work has indicated that LXTYF had great pharmacology efficacy on AICH by in vivo and in vitro experiment [14].

In our research, network pharmacology method is applied to predict active components, targets, and pathways of LXTYF to alleviate AICH. TCMs provide multiple components, targets, and pathways, also with similar characteristics with systems biology and network pharmacology. This indicates that we can use the approach of the network pharmacology to deeply investigate the mechanisms of TCMs to alleviate AICH [15]. Further, pivotal human genes and pathways were verified after the conversion of rat transcriptomics RNA-seq data (Figure 1). Especially, in the animal experiment, the commonly used AICH model of spontaneous hypertension rats (SHRs) induced by collagenase was made, and LXTYF administration was then conducted for RNA-seq.
This study is aimed at digging into the system pharmacological mechanism of Liangxue Tongyu Formula (LXTYF) on treating intracerebral hemorrhage via network pharmacology method and RNA sequencing data and providing notable clues for further experimental validation.

\section{Materials and Methods}

2.1. Herb Formulation Ingredients Collection. The chemical ingredients of six botanical medicinal materials in LXTYF were obtained from Traditional Chinese Medicine Systems Pharmacology Database and Analysis Platform (TCMSP) (http://lsp. http://nwu.edu.cn/tcmsp.php) [16]. TCMSP is a distinctive platform for TCM pharmacology, able to provide herbal information, ADME data for the screening process and related targets of each chemical ingredient. The chemical ingredients of the other two animal medicines in LXTYF were derived from BATMAN-TCM (https://bionet.ncpsb .org/batman-tcm) [17]. BATMAN-TCM is the first online Bioinformatics Analysis Tool for the Molecular mechanism of TCM, which can provide the active ingredients and ranked target prediction of animal medicines for analysis.

2.2. Active Ingredients Screening. The active ingredients from herbs of LXTYF were filtered by OB and DL. OB represents the relative amount of a drug absorbed into the blood circulation, and DL is used to describe pharmaceutical properties of compounds $[18,19]$. Based on literature and suggestions in TCMSP, $\mathrm{OB} \geq 30 \%$ and $\mathrm{DL} \geq 0.18$ were selected as a screening threshold [20-22]. Ingredients that meet the above rules will be preserved for further analysis. Moreover, several active ingredients of Dihuang, Sanqi, and Shichangpu with important pharmacological effects were added into the compound database for analysis based on the literature about chemical composition and the Chinese Pharmacopoeia 2015.

2.3. Active Ingredient-Associated Target Prediction. TCMSP and BATMAN-TCM databases are integrated to collect known targets of active ingredients in LXTYF. The target names were obtained from the TCMSP and then converted into Uniprot ID in the "Retrieve/ID Mapping" tool of Uniprot (Universal Protein Resource) database (http://www .uniprot.org/), which is specialized for protein information [23]. To analyze those active components that have unknown target proteins, the following databases were used. PubChem is the world's largest collection of chemical information, in which name, molecular formula, structure, physical properties, and so on can be found [24]. Open Babel toolkit is a toolbox transforming multiple chemical file formats $[25,26]$. With the help of these two databases, SMILES formats of those active components were converted. SwissTargetPrediction is a web-based tool used to predict the most possible targets for small molecules. The target predictions are achieved through reverse screening based on the similarity principle [27]. The top 15 potential targets of each active component were predicted from the SwissTargetPrediction database.

2.4. ICH-Associated Target Prediction. Different genes linked to $\mathrm{ICH}$ were collected from three existing resources with Medical Subject Heading (MeSH) "Cerebral Hemorrhage". 


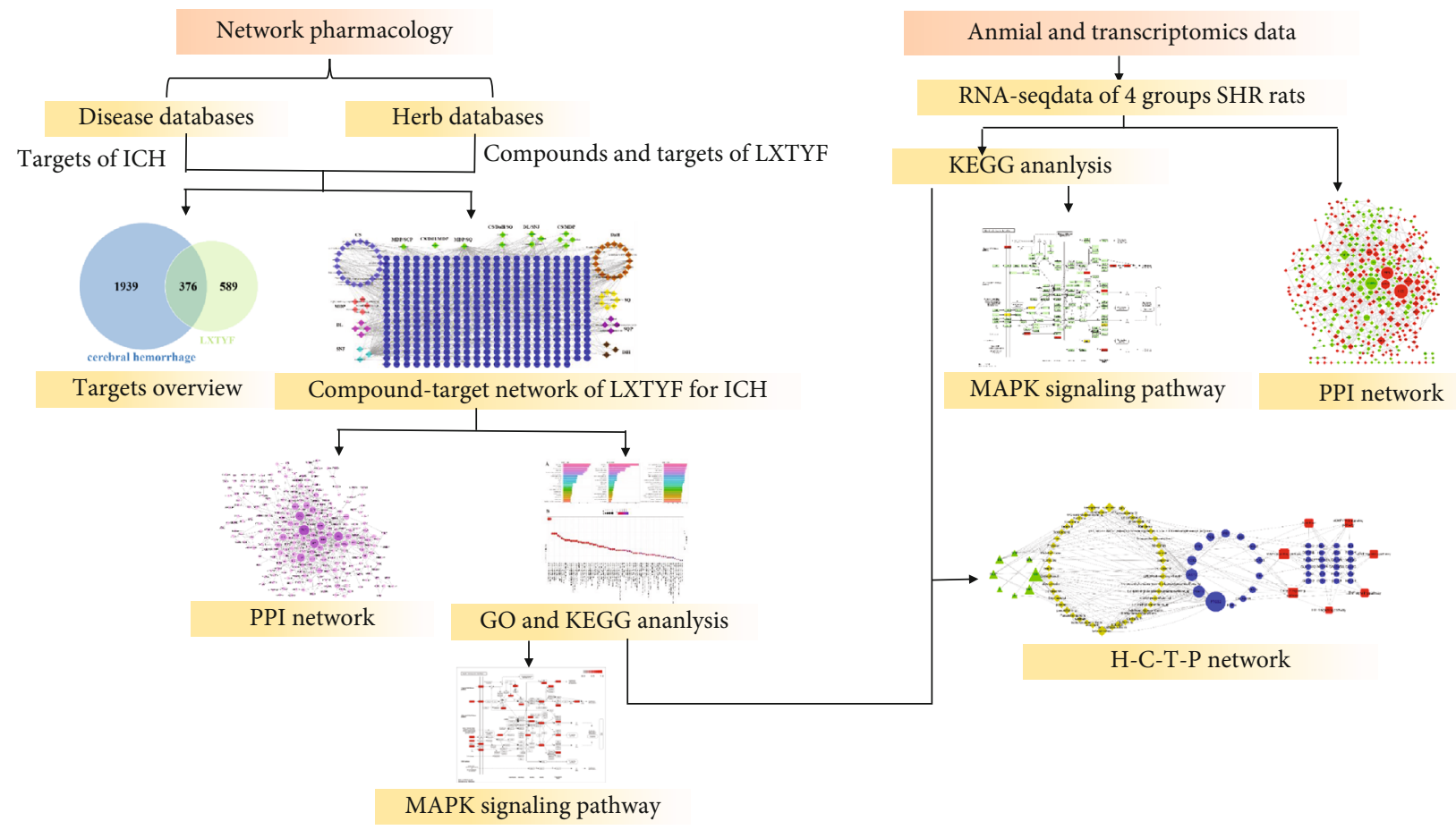

FIgURE 1: Outline of the study. Network pharmacology method and transcriptomics data were applied to explore the LXTYF effect on AICH.

(1) GAD (http://geneticassociationdb.nih.gov/), a public genetic association database serving for human genetic diseases based on integrated datas of more than 5000 published studies [28]. (2) Genecards (https://www.genecards.org/), an automatically mined database of human genes and a very wide range of resources for gene-centric information in the human genome [29, 30]. (3) DisGeNET (http://www .disgenet.org/web/DisGeNET), which integrates data from animal models, GWAS catalogues, expertly curated repositories, and the scientific literature, constructing a comprehensive platform studying human disease-associated genes and variants [31].

2.5. Network Construction and Key Nodes Analysis. Putative targets of LXTYF for the treatment of AICH were obtained based on the interaction data between active ingredientassociated targets and $\mathrm{ICH}$-associated targets, and Cytoscape software (Version 3.2.1) was utilized to visualize the network for AICH-associated-active ingredients and targets. Degree and betweenness centrality are important topological parameters of networks and were calculated in Cytoscape to measure the topological importance of target and compound nodes [32-34]. The nodes were identified as key targets and key chemical ingredients according to the "Degree" and "Betweenness Centrality" values, which were larger than the average degree and average betweenness centrality of all nodes in the network.

2.6. PPI Network Construction. Proteins are rare to achieve specific functions alone and tend to form macromolecular complexes by interaction in a single cell to complete biological functions [34]. So, it is necessary to analyze the interactions in the proteins-proteins interaction (PPI) network.
STRING 11.0 (https://string-db.org/) is a database with integrated data from several active interaction sources such as text mining, experiments, databases, and coexpression. It uploads gene datasets as input and visualizes them in a network for the next analysis [35]. In this study, STRING was used to analyze the interactions between targets (cut - off critical level of high interaction score confidence $=$ 0.95) of LXTYF for AICH.

2.7. Gene Ontology and Pathway Enrichment. The ClusterProfiler package installed in $\mathrm{R}$ offers the groupGO method to classify genes [36]. There are three aspects associated with GO terms as follows: cellular components (CC), molecular functions (MF), and biological processes (BP). Also, by hypergeometric distribution, enrichKEGG function is provided to proceed with enrichment test for KEGG pathways. $p$.adjust values are also estimated to prevent a high false discovery rate (FDR). Pathways that the $p$.adjust $\leq 0.05$ were reserved to analyze.

2.8. Animals and Reagents. Eight crude herbs of LXTYF were purchased from Tongrentang Chinese Medicine Company (Since 1669). Spontaneous hypertension rats (SHRs) were purchased from Beijing Vital River Laboratory Animal Technology Co., Ltd., and animal license number was SCXK (Beijing) 2016-0006. Collagenase type VII was purchased from Sigma-Aldrich (St Louis, MO, USA). Brain stereotaxic instruments and electric drills were bought from Yuyan Instruments Co., Ltd. (Shanghai, China). TJ-1A microinjection pump was purchased from Lange Constant Flow Pump Co., Ltd. (Baoding, China). Microneedle was purchased from Gaoge Industry \& Trade Co., Ltd. (Shanghai, China). Chloral hydrate and penicillin were bought from Sinopharm 
Chemical Reagent Co., Ltd. (Shanghai, China). TRIzol ${ }^{\circledR}$ Reagent and TBS380 Picogreen were purchased from Invitrogen (Carlsbad, CA, USA). Magnetic frame Ribo-Zero Magnetic kit was bought from EpiCentre (USA). TruSeqTM Stranded Total RNA Library Prep Kit, UNG enzyme, HiSeq 4000 PE Cluster Kit, and HiSeq 4000 SBS Kit (300cycles) were purchased from Illumina (San Diego, CA, USA). Certified Low Range Ultra Agarose was purchased from Bio-Rad (Hercules, CA, USA).

\subsection{LXTYF Preparation and Animal Administration. LXTYF} consisted of Cornu Bubali (SNJ), Rheum palmatum L. (DaH), Rehmannia glutinosa Libosch. (DiH), Panax notoginseng (Burk.) F.H.Chen (SQ), Radix paeoniae rubra (CS), Moutan Cortex (MDP), Pheretima aspergillum (DL), and Acori Tatarinowii Rhizoma (SCP). The crude herbs mixed in the weight ratio of $30: 10: 20: 5: 15: 10: 10: 10$ and extracted by means of reflux in ethanol and water for 3 hours. LXTYF decoction was prepared with $8: 1$ water/oil.

All procedures concerning animals in this study were approved by the ethics committee of Nanjing University of Chinese Medicine. Spontaneous hypertension rats (SHRs) were randomly assigned into cages and adapted for experiment environment in the SPF-room for one week, with food and water regularly and temperature at $20-25^{\circ} \mathrm{C}$. 20 male SHR rats (230-260 g) were divided into four groups randomly for modeling and experiments: control group (CG), control group with LXTYF treatment (CGT), ICH-model group (MG), and AICH model with LXTYF-treatment group (MGT), with 5 SHR rats in each group. Rats in CG and MG were given physiological saline, while CGT and MGT rats received intragastric administration of LXTYF decoction for 5 days. $12 \mathrm{~h}$-fasting-rats in MG and MGT were made AICH models by using $0.12 \mathrm{U}$ collagenase of $2 \mu \mathrm{l}$ and brain stereotaxic instrument. According to our previous study, the middle dose of $11.55 \mathrm{~g} / \mathrm{kg}$ of LXTYF has the best efficiency on survival time and rate for rats with AICH. So, all the dosage of the following administration was $11.55 \mathrm{~g} / \mathrm{kg}$. After 24 hours of modeling, rats were sacrificed by decapitation, and brain tissue of the rat was quickly obtained from where the microinjector was inserted. The specimens of brain tissues were frozen in liquid nitrogen and stored in $-80^{\circ} \mathrm{C}$ refrigerator for RNA-seq.

2.10. Sequencing for Differentially Expressed $m R N A$. The specimens of brain tissues were obtained from the rats (4 groups with 3 replicates). Total RNA was extracted from brain tissue specimens and tested for its concentration and purity. $5 \mu \mathrm{g}$ of rat total RNA was started, and the concentration was $\geq 250 \mathrm{ng} / \mathrm{ml}$. OD260/280 was between 1.8 and 2.2. After removing the rRNA, indexed libraries were prepared from purified RNA with TruSeqTM Stranded Total RNA Library Prep Kit (Illumina) according to the manufacturer's instructions. Randomly interrupted with metal ions, $2000 \mathrm{bp}$ mRNA fragments were obtained. The first strand cDNA synthesis was performed according to the mRNA fragments as described in the Illumina kit. The second strand cDNA synthesis was modified by exchanging dTTP with dUTP. After adding end repair mix to double-stranded
cDNA, blunt ends were performed, and A bases were added to attach the Y-shaped adapters. The UNG enzyme digested the second-strand CDNA, and the library only contained the first-strand cDNA. Bridge PCR amplification was performed on cBot, and $2 * 150 \mathrm{bp}$ sequencing was performed on Illumina Hiseq. Further details can be found in the references $[37,38]$.

2.11. Verification of Related $m R N A$ s and Pathways. With the thresholds of $\geq 2$-fold and the $p$ value of $\leq 0.05$ via edgeR (the Empirical Analysis of Digital Gene Expression in R, v3.2.4) software, differentially expressed mRNAs were identified especially the MGT and MG. Rat genes were converted into human genes based on homology mapping through the Biomart package in R. Compared with the putative targets that LXTYF treats for AICH, PPI network of verified genes and other genes was constructed in STRING and Cytoscape software.

KEGG orthology-based annotation system (KOBAS) is a web server for annotation and identification of enriched pathways and diseases [39]. In our research, enrichment analysis of the differentially expressed mRNAs was performed using KOBAS 3.0 (http://kobas.cbi.pku.edu.cn/ anno_iden.php). In the KEGG database, Fisher's exact test was used as a statistical method, and Benjamini and Hochberg was used as an FDR correction method. Enriched pathways that $p$ value $\leqq 0.05$ were identified compared with former pathway enrichment prediction.

2.12. Herb-Compound-Target-Pathway(H-C-T-P) Network Construction. Compared the pathways enriched by the network pharmacology method with those enriched by RNAseq data, the common and most likely pathways are obtained. Differentially expressed genes related to common pathways are achieved from the RNA-seq data. The verified generelated compounds and herbs are found from the collected network pharmacology datas, connections among them were shown in the Herb-Compound-Target-Pathway(H-C-T-P) network and visualized in Cytoscape software.

\section{Results}

3.1. Active Ingredients of LXTYF. A total of 517 compounds of 8 herbs in LXTYF were collected from TCMSP and BATMAN-TCM database after removing duplicate items, including 119 compounds from CS, 92 compounds from $\mathrm{DaH}, 76$ compounds from $\mathrm{DiH}, 119$ compounds from SQ, 55 compounds from MDP, 105 compounds from SCP, 6 compounds from SNJ, and 8 compounds from DL (S1 Table).

Considering the drug formation of the compound and metabolism after being absorbed into the human body, 81 active ingredients of LXTYF filtered by OB and DL were screened out from the 517 compounds with ADME parameters as shown in Table 1. Specifically, there were 30 active ingredients in CS, 19 active ingredients in $\mathrm{DaH}, 12$ active ingredients in MDP, 12 active ingredients in SQ, 7 active ingredients in DL, 6 active ingredients in SNJ, 5 active ingredients in $\mathrm{DiH}$, and 5 active ingredients in SCP. Among them, 
TABLE 1: Description of ADME parameters of LXTYF active ingredients.

\begin{tabular}{|c|c|c|c|c|c|}
\hline Herb & Mol ID & Molecule name & MW & $\begin{array}{l}\text { OB } \\
(\%)\end{array}$ & DL \\
\hline CS & MOL001002 & Ellagic acid & 302.2 & 43.06 & 0.43 \\
\hline CS & MOL001918 & Paeoniflorgenone & 318.35 & 87.59 & 0.37 \\
\hline CS & MOL001924 & Paeoniflorin & 480.51 & 53.87 & 0.79 \\
\hline CS & MOL002714 & Baicalein & 270.25 & 33.52 & 0.21 \\
\hline CS & MOL002776 & Baicalin & 446.39 & 40.12 & 0.75 \\
\hline CS & MOL002883 & Ethyl oleate (NF) & 310.58 & 32.4 & 0.19 \\
\hline CS & MOL004355 & Spinasterol & 412.77 & 42.98 & 0.76 \\
\hline CS & MOL005043 & Campest-5-en-3beta-ol & 400.76 & 37.58 & 0.71 \\
\hline CS & MOL006992 & $(2 \mathrm{R}, 3 \mathrm{R})-4$-methoxyl-distylin & 318.3 & 59.98 & 0.3 \\
\hline CS & MOL006999 & Stigmast-7-en-3-ol & 414.79 & 37.42 & 0.75 \\
\hline CS & MOL001921 & Lactiflorin & 462.49 & 49.12 & 0.8 \\
\hline CS & MOL006990 & (1S,2S,4R)-trans-2-hydroxy-1,8-cineole-B-D-glucopyranoside & 332.44 & 30.25 & 0.27 \\
\hline CS & MOL006994 & 1-O-beta-d-glucopyranosyl-8-o-benzoylpaeonisuffrone_qt & 302.35 & 36.01 & 0.3 \\
\hline CS & MOL006996 & 1-O-beta-d-glucopyranosylpaeonisuffrone_qt & 332.38 & 65.08 & 0.35 \\
\hline CS & MOL007004 & Albiflorin & 480.51 & 30.25 & 0.77 \\
\hline CS & MOL007005 & Albiflorin_qt & 318.35 & 48.7 & 0.33 \\
\hline CS & MOL007008 & 4-Ethyl-paeoniflorin_qt & 332.38 & 56.87 & 0.44 \\
\hline CS & MOL007012 & 4-O-methyl-paeoniflorin_qt & 332.38 & 56.7 & 0.43 \\
\hline CS & MOL007014 & 8-Debenzoylpaeonidanin & 390.43 & 31.74 & 0.45 \\
\hline CS & MOL007016 & Paeoniflorigenone & 318.35 & 65.33 & 0.37 \\
\hline CS & MOL007018 & 9-Ethyl-neo-paeoniaflorin A_qt & 334.4 & 64.42 & 0.3 \\
\hline CS & MOL007022 & Evofolin B & 318.35 & 64.74 & 0.22 \\
\hline CS & MOL007025 & Isobenzoylpaeoniflorin & 584.62 & 31.14 & 0.54 \\
\hline $\mathrm{DaH}$ & MOL000096 & (-)-Catechin & 290.29 & 49.68 & 0.24 \\
\hline $\mathrm{DaH}$ & MOL000471 & Aloe-emodin & 270.25 & 83.38 & 0.24 \\
\hline $\mathrm{DaH}$ & MOL000554 & Gallic acid-3-O-(6'-O-galloyl)-glucoside & 484.4 & 30.25 & 0.67 \\
\hline $\mathrm{DaH}$ & MOL002235 & EUPATIN & 360.34 & 50.8 & 0.41 \\
\hline $\mathrm{DaH}$ & MOL002259 & Physciondiglucoside & 608.6 & 41.65 & 0.63 \\
\hline $\mathrm{DaH}$ & MOL002268 & Rhein & 284.23 & 47.07 & 0.28 \\
\hline $\mathrm{DaH}$ & MOL002280 & Torachrysone-8-O-beta-D-(6' -oxayl)-glucoside & 480.46 & 43.02 & 0.74 \\
\hline $\mathrm{DaH}$ & MOL002281 & Toralactone & 272.27 & 46.46 & 0.24 \\
\hline $\mathrm{DaH}$ & MOL002297 & Daucosterol_qt & 386.73 & 35.89 & 0.7 \\
\hline $\mathrm{DaH}$ & MOL002251 & Mutatochrome & 552.96 & 48.64 & 0.61 \\
\hline $\mathrm{DaH}$ & MOL002260 & Procyanidin B-5,3'-O-gallate & 730.67 & 31.99 & 0.32 \\
\hline $\mathrm{DaH}$ & MOL002276 & Sennoside E_qt & 524.5 & 50.69 & 0.61 \\
\hline $\mathrm{DaH}$ & MOL002288 & Emodin-1-O-beta-D-glucopyranoside & 432.41 & 44.81 & 0.8 \\
\hline $\mathrm{DaH}$ & MOL002293 & Sennoside D_qt & 524.5 & 61.06 & 0.61 \\
\hline $\mathrm{DaH}$ & MOL002303 & Palmidin A & 510.52 & 32.45 & 0.65 \\
\hline $\mathrm{DaH}$ & MOL000472 & Emodin* & 270.25 & 24.4 & 0.24 \\
\hline $\mathrm{DaH}$ & MOL001729 & Crysophanol* $^{*}$ & 254.25 & 18.64 & 0.21 \\
\hline $\mathrm{DaH}$ & MOL000476 & Physcion* & 284.28 & 22.29 & 0.27 \\
\hline $\mathrm{DiH}$ & MOL002819 & Catalpol* $^{*}$ & 362.37 & 5.07 & 0.44 \\
\hline $\mathrm{DiH}$ & MOL003333 & Acteoside $^{*}$ & 624.65 & 2.94 & 0.62 \\
\hline $\mathrm{DiH}$ & MOL000732 & Stachyose* & 666.66 & 3.25 & 0.59 \\
\hline MDP & MOL000211 & Mairin & 456.78 & 55.38 & 0.78 \\
\hline MDP & MOL007374 & 5-[[5-(4-Methoxyphenyl)-2-furyl] 'methylene]barbituric acid & 312.3 & 43.44 & 0.3 \\
\hline MDP & MOL007369 & 4-O-methylpaeoniflorin_qt & 332.38 & 67.24 & 0.43 \\
\hline
\end{tabular}


TABLE 1: Continued.

\begin{tabular}{|c|c|c|c|c|c|}
\hline Herb & Mol ID & Molecule name & MW & $\begin{array}{l}\text { OB } \\
(\%)\end{array}$ & $\mathrm{DL}$ \\
\hline MDP & MOL007382 & Mudanpioside-h_qt 2 & 336.37 & 42.36 & 0.37 \\
\hline MDP & MOL007384 & Paeonidanin_qt & 330.41 & 65.31 & 0.35 \\
\hline SQ & MOL001494 & Mandenol & 308.56 & 42 & 0.19 \\
\hline SQ & MOL001792 & $\mathrm{DFV}$ & 256.27 & 32.76 & 0.18 \\
\hline SQ & MOL002879 & Diop & 390.62 & 43.59 & 0.39 \\
\hline SQ & MOL005344 & Ginsenoside rh2 & 622.98 & 36.32 & 0.56 \\
\hline SQ & MOL007475 & Ginsenoside f2 & 785.14 & 36.43 & 0.25 \\
\hline SQ & MOL007476 & Ginsenoside $r b 1^{*}$ & $1,109.46$ & 6.29 & 0.04 \\
\hline SQ & MOL005338 & Ginsenoside $\mathrm{Re}^{*}$ & 947.3 & 4.27 & 0.12 \\
\hline SQ & MOL005341 & Sanchinoside $\mathrm{C} 1^{*}$ & 801.14 & 10.04 & 0.28 \\
\hline SQ & MOL007487 & Notoginsenoside $r 1^{*}$ & 933.27 & 5.43 & 0.13 \\
\hline SCP & MOL001944 & Marmesin & 246.28 & 50.28 & 0.18 \\
\hline SCP & MOL003542 & 8-Isopentenyl-kaempferol & 354.38 & 38.04 & 0.39 \\
\hline SCP & MOL003576 & $\begin{array}{c}\text { (1R,3aS,4R,6aS)-1,4-bis(3,4-dimethoxyphenyl)-1,3,3a,4,6,6a-hexahydrofuro[4,3- } \\
\text { c]furan }\end{array}$ & 386.48 & 52.35 & 0.62 \\
\hline SCP & MOL003578 & Cycloartenol & 426.8 & 38.69 & 0.78 \\
\hline $\mathrm{MDP} / \mathrm{SQ}$ & MOL000098 & Quercetin & 302.25 & 46.43 & 0.28 \\
\hline $\mathrm{CS} / \mathrm{DaH} / \mathrm{SQ}$ & MOL000358 & Beta-sitosterol & 414.79 & 36.91 & 0.75 \\
\hline CS/DiH/MDP & MOL000359 & Sitosterol & 414.79 & 36.91 & 0.75 \\
\hline MDP/SCP & MOL000422 & Kaempferol & 286.25 & 41.88 & 0.24 \\
\hline CS/DiH/SQ & MOL000449 & Stigmasterol & 412.77 & 43.83 & 0.76 \\
\hline CS/MDP & MOL000492 & $(+)$-Catechin & 290.29 & 54.83 & 0.24 \\
\hline $\mathrm{CS} / \mathrm{MDP}$ & MOL000874 & Paeonol $^{*}$ & 166.19 & 28.79 & 0.04 \\
\hline CS/MDP & MOL001925 & Paeoniflorin_qt & 318.35 & 68.18 & 0.4 \\
\hline CS/MDP & MOL007003 & Benzoyl paeoniflorin & 584.62 & 31.14 & 0.54 \\
\hline $\mathrm{DL}$ & -- & Xanthine & -- & -- & - - \\
\hline DL & -- & Guanosine & -- & -- & - - \\
\hline DL & -- & Xanthinin & - - & -- & - - \\
\hline DL & -- & Hyrcanoside & -- & -- & - - \\
\hline SNJ & -- & Arginine & -- & -- & - - \\
\hline SNJ & -- & Aspartic acid & -- & - & - - \\
\hline SNJ & -- & Alanine & -- & - & - - \\
\hline $\mathrm{DL} / \mathrm{SNJ}$ & -- & 4-Guanidino-1-butanol & - - & - & - - \\
\hline $\mathrm{DL} / \mathrm{SNJ}$ & -- & Cholesterol & - - & - - & - - \\
\hline $\mathrm{DL} / \mathrm{SNJ}$ & -- & Guanidine & - - & - & - - \\
\hline
\end{tabular}

“*” means the active ingredients retrieved by literature mining that cannot fit the earlier screening criteria but with important pharmacological effects. “-_, means the active ingredients collected from the BAT-MAN database with no ADME parameters. SNJ: Cornu Bubali; DaH: Rheum palmatum L.; DiH: Rehmannia glutinosa Libosch.; SQ: Panax notoginseng (Burk.) F.H.Chen; CS: Radix paeoniae rubra; MDP: Moutan Cortex; DL: Pheretima aspergillum; SCP: Acori Tatarinowii Rhizoma.

beta-sitosterol, sitosterol, and stigmasterol are shared by three herbs. Quercetin, kaempferol, (+)-catechin, paeonol, paeoniflorin_qt, benzoyl paeoniflorin, 4-guanidino-1-butanol, cholesterol, and guanidine are common active ingredients of two herbs.

3.2. Putative Targets of LXTYF for the Treatment of AICH and Network Construction. On the basis of the 81 active ingredients of LXTYF above, 965 targets were collected from two databases with no duplicate values. Among them, 874 targets of the active ingredients were obtained from the TCMSP (S2 Table), and 149 targets of those active components that have unknown target protein were predicted from the SwissTargetPrediction database (S3 Table). From the GAD, Genecards, and DisGeNET database, 2315 targets relating to $\mathrm{ICH}$ were collected (S4 Table). After overlapping the 2315 targets with former 965 targets, 376 targets of LXTYF for treating AICH were obtained as shown in Figure 2(a). At the help of Cytoscape, a putative component-target network composed of 452 nodes and 890 


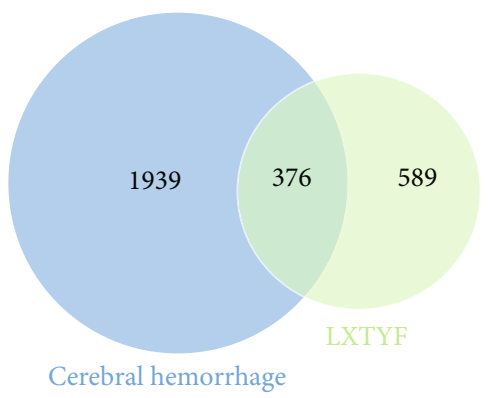

(a)

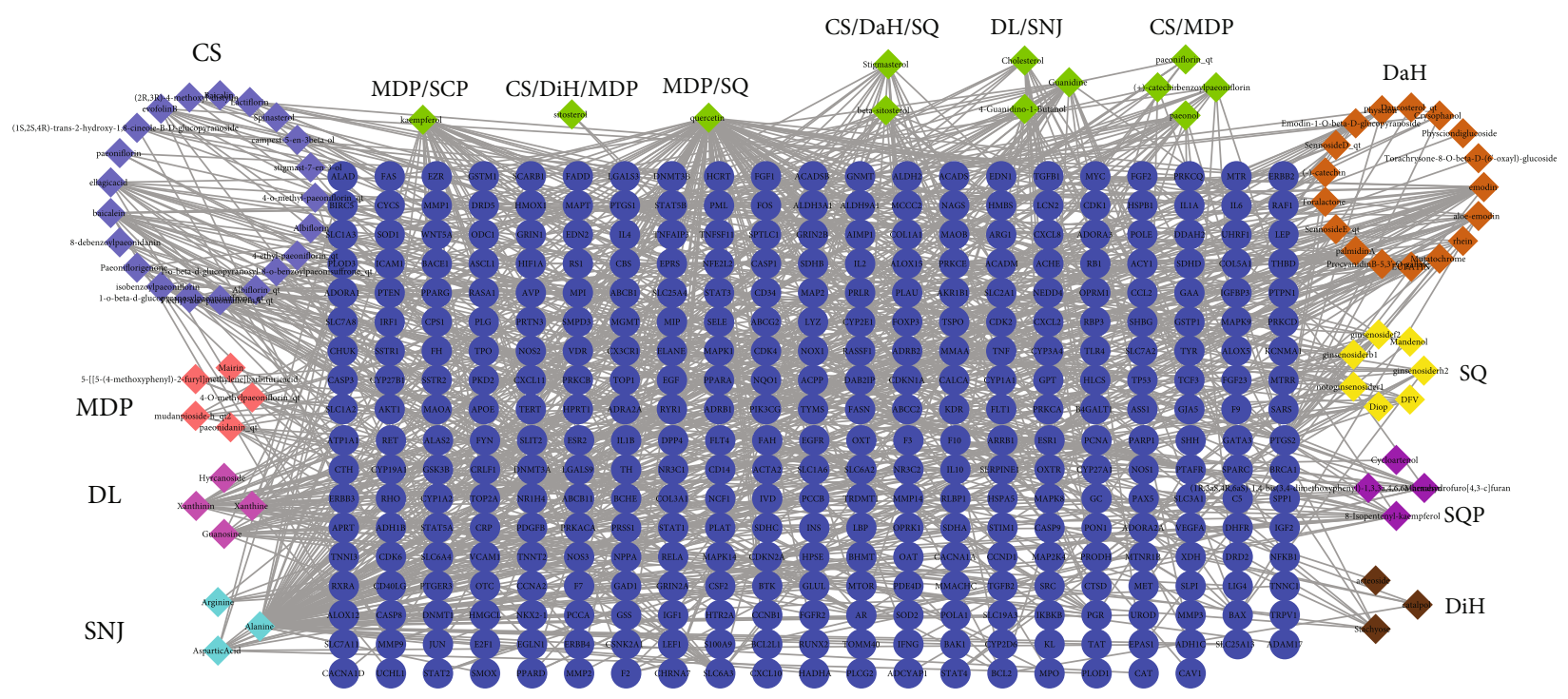

(b)

FIGURE 2: Network construction. (a) Veen diagram describing targets distribution of LXTYF and ICH. (b) The network of Putative Component-Target. Blue ellipses refer to 376 targets of LXTYF for AICH treatment. Different color triangles represent 76 active components in LXTYF. In particular, green triangles stand for common compounds of three and two herbs.

edges were constructed based on 376 targets and the corresponding 76 components as Figure 2(b).

\subsection{Network Analysis and Key Nodes Screening. Topological} analysis is an effective approach to evaluate the abovementioned component-target network based on the degree and betweenness centrality parameters (S5 Table for details). The following are the obtained results of the network topological analysis: network heterogeneity (2.144), network density (0.009), and shortest paths (202052, 99\%). In the component-target network, the first three targets are PTGS2 $($ degree $=24)$, PTGS1 $($ degree $=21)$, and ESR1 $($ degree $=17)$. The first three components are quercetin (degree $=112$ ), alanine (degree $=104)$, and kaempferol (degree $=39)$, which are, respectively, from MDP/SQ, SNJ, and MDP/SCP.

From an in-depth analysis of the complete network above, key components and targets with imporant biological functions can be identified. The nodes (compound and target) whose degrees and betweeness centralities are both larger than the average ones were identified as key targets and chemical ingredients. The average degree of the nodes is 3.938 , and 122 nodes are larger than this number. The average betweenness centrality of nodes is 0.006549 and 85 nodes are larger than it. In general, 74 key nodes (35 key components from 76 components and 39 key targets from 376 targets) were obtained, which was shown in Table 2. They were extracted and the connections of the keycompound-target network were displayed in S1 Fig. The first 3 key targets are PTGS2, PTGS1, and AR, and the first 3 key components are quercetin, kaempferol, and emodin, which may all have a great effect on AICH treatment.

3.4. PPI Network Construction. For analysis of the proteinsproteins interaction network of LXTYF for AICH, STRING 11.0 was used to analyze the targets of LXTYF (Results in S6 Table). Apart from the targets independent of the network and the confidence level of interaction score less than 0.95 , 275 related targets (from 376 targets) and 850 interactions were included in the network visualized by Cytoscape in Figure 3. The importance of key proteins was then evaluated in light of the degree of the nodes exported from the STRING database. It is clear that the STAT3 (degree $=45$ ) is much larger than other nodes in the network (Avg.numbers of neighbors $=6.182$ ), followed by TP53, AKT1, SRC, JUN, TNF, and MAPK1 (degree values larger than 30 ). The topological parameters of the PPI network are shown in S7 Table. 
TABLE 2: Key nodes of component-target network.

\begin{tabular}{|c|c|c|c|}
\hline Herb & Node & Degree & $\begin{array}{c}\text { Betweenness } \\
\text { centrality }\end{array}$ \\
\hline MDP/SQ & Quercetin & 112 & 0.36958975 \\
\hline SNJ & Alanine & 104 & 0.41797809 \\
\hline MDP/SCP & Kaempferol & 39 & 0.06875802 \\
\hline $\mathrm{DaH}$ & Emodin & 29 & 0.04544646 \\
\hline DL/SNJ & Guanidine & 27 & 0.08907938 \\
\hline $\mathrm{DL}$ & Xanthinin & 25 & 0.08396791 \\
\hline $\mathrm{DL}$ & Xanthine & 24 & 0.08789741 \\
\hline CS & Baicalein & 23 & 0.02318204 \\
\hline CS/MDP & Paeonol & 20 & 0.02429964 \\
\hline $\mathrm{CS} / \mathrm{DaH} / \mathrm{SQ}$ & Beta-sitosterol & 20 & 0.02791937 \\
\hline $\mathrm{DL}$ & Guanosine & 19 & 0.06005265 \\
\hline SCP & 8-Isopentenyl-kaempferol & 18 & 0.02608771 \\
\hline $\mathrm{DaH}$ & Aloe-emodin & 18 & 0.01960079 \\
\hline $\mathrm{CS} / \mathrm{DaH} / \mathrm{SQ}$ & Stigmasterol & 18 & 0.03894191 \\
\hline DL/SNJ & Cholesterol & 16 & 0.05001097 \\
\hline CS & Ellagicacid & 15 & 0.01790131 \\
\hline SNJ & Aspartic acid & 13 & 0.03120851 \\
\hline SCP & Marmesin & 13 & 0.0165369 \\
\hline DL/SNJ & 4-Guanidino-1-butanol & 12 & 0.03705792 \\
\hline $\mathrm{DaH}$ & EUPATIN & 12 & 0.01168363 \\
\hline CS/MDP & Benzoylpaeoniflorin & 12 & 0.01694236 \\
\hline CS & Albiflorin_qt & 12 & 0.0149345 \\
\hline SQ & Ginsenoside rb1 & 11 & 0.01531869 \\
\hline SQ & Ginsenoside rh2 & 10 & 0.01552107 \\
\hline CS & $\begin{array}{l}\text { 1-O-beta-d-glucopyranosyl- } \\
\text { 8-o-benzoylpaeonisuffrone_- } \\
\text { qt }\end{array}$ & 10 & 0.00756521 \\
\hline CS & Evofolin B & 10 & 0.01443392 \\
\hline SQ & Notoginsenoside r1 & 9 & 0.00950678 \\
\hline SQ & Ginsenoside f2 & 9 & 0.0087669 \\
\hline $\mathrm{DaH}$ & Mutatochrome & 9 & 0.0191967 \\
\hline $\mathrm{DaH}$ & $\begin{array}{c}\text { Procyanidin B-5, } 3^{\prime}-\mathrm{O}- \\
\text { gallate }\end{array}$ & 9 & 0.02300249 \\
\hline CS & Albiflorin & 9 & 0.00983057 \\
\hline $\mathrm{DiH}$ & Stachyose & 8 & 0.00668771 \\
\hline $\mathrm{DaH}$ & Palmidin A & 6 & 0.010557 \\
\hline CS & 8-Debenzoylpaeonidanin & 4 & $1.33 E-02$ \\
\hline CS & Paeoniflorin & 4 & 0.00892285 \\
\hline Target & PTGS2 & 24 & 0.03392333 \\
\hline Target & PTGS1 & 21 & 0.0243207 \\
\hline Target & ESR1 & 17 & 0.04743098 \\
\hline Target & MAPT & 15 & 0.02405041 \\
\hline Target & PRKACA & 15 & 0.0125587 \\
\hline Target & $\mathrm{AR}$ & 14 & 0.0546651 \\
\hline Target & PTAFR & 14 & 0.01000789 \\
\hline Target & PGR & 13 & 0.04286906 \\
\hline Target & SLC6A2 & 13 & 0.01380599 \\
\hline Target & SLC6A3 & 12 & 0.00778408 \\
\hline
\end{tabular}

TABLE 2: Continued.

\begin{tabular}{|c|c|c|c|}
\hline Herb & Node & Degree & $\begin{array}{c}\text { Betweenness } \\
\text { centrality }\end{array}$ \\
\hline Target & ADRB2 & 9 & 0.06270829 \\
\hline Target & BAX & 8 & 0.01594706 \\
\hline Target & ESR2 & 8 & 0.01052083 \\
\hline Target & F10 & 8 & 0.0115522 \\
\hline Target & PRKCA & 8 & 0.07827366 \\
\hline Target & RXRA & 8 & 0.01249876 \\
\hline Target & TOP2A & 8 & 0.01135885 \\
\hline Target & CDK2 & 7 & 0.00778363 \\
\hline Target & F7 & 7 & 0.00821191 \\
\hline Target & TNF & 7 & 0.01279629 \\
\hline Target & $\mathrm{F} 2$ & 6 & 0.00701019 \\
\hline Target & IL1B & 6 & 0.06205608 \\
\hline Target & NOS2 & 6 & 0.01729864 \\
\hline Target & PRKCD & 6 & 0.00681975 \\
\hline Target & VEGFA & 6 & 0.01754771 \\
\hline Target & AKR1B1 & 5 & 0.02396875 \\
\hline Target & JUN & 5 & 0.02537208 \\
\hline Target & MMP9 & 5 & 0.00770693 \\
\hline Target & NOS3 & 5 & 0.02534139 \\
\hline Target & NR3C2 & 5 & 0.0069026 \\
\hline Target & STAT1 & 5 & 0.01728485 \\
\hline Target & ADORA1 & 4 & 0.04165433 \\
\hline Target & ADRA2A & 4 & 0.01897542 \\
\hline Target & CYP1A1 & 4 & 0.01430838 \\
\hline Target & GSTP1 & 4 & 0.02559893 \\
\hline Target & HIF1A & 4 & 0.02506214 \\
\hline Target & PPARD & 4 & 0.02353186 \\
\hline Target & TGFB1 & 4 & $1.03 E-02$ \\
\hline Target & VDR & 4 & $8.61 E-03$ \\
\hline
\end{tabular}

3.5. Gene Ontology and Pathway Enrichment for Putative Targets. For further study about the putative targets of LXTYF on AICH action, Clusterprofiler package in R was used for analysis due to its higher classification and enrichment accuracy, as well as the visualization module for the results [36]. 376 targets above were applied to run the package for gene classification and KEGG enrichment analysis $(F D R \leq 0.05)$. Figure $4(a)$ depicted the top $20 \mathrm{GO}$ terms of biological processes (BP), molecular functions (MF), and cellular components (CC), respectively. Results covered that 376 targets of LXTYF for treatment on AICH in BP were mainly associated with positive regulation of the cellular process, signal transduction, and cellular macromolecule metabolic process. In the aspect of MF, the targets mainly participated in the process of protein binding, ion binding, and cyclic compound binding. Obviously, the cellular components mainly occur in the cytoplasm, intracellular organelle, and nucleus.

Figure 4(b) showed 72 enriched KEGG pathways identified using the Clusterprofiler package. The top 5 enriched 


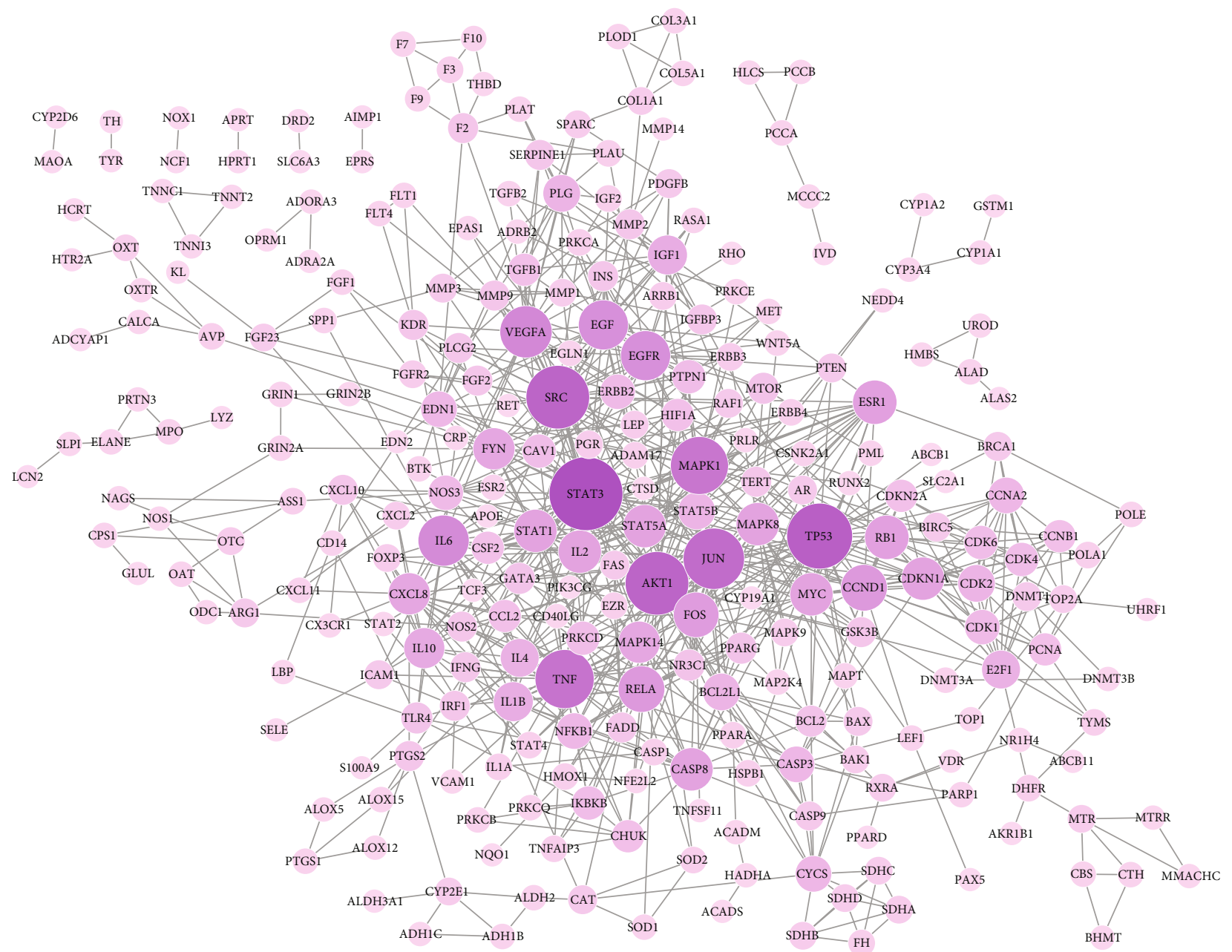

FIGURE 3: PPI network of LXTYF targets for treating intracerebral hemorrhage (larger degree of the target node with larger and deeper color).

pathways were MAPK signaling pathway, PI3K-Akt signaling pathway, TNF signaling pathway, cAMP signaling pathway, and Ras signaling pathway, with $49,49,33,33$, and 33 genes, respectively. See S8 Table for results of putative targets for KEGG pathway enrichment analysis. The most highly enriched pathways related to LXTYF for AICH were the MAPK signaling pathway and the PI3K-Akt signaling pathway. MAPK signaling pathway was shown in Figure 5.

3.6. Identification of Mutually Expressed mRNAs. With a fold-change cutoff of $2(p \leq 0.05)$, the analysis of differently expressed genes among four groups from the RNA-seq data was shown in Table 3 ( 3 duplicate values in each group). 766 differentially expressed genes between MG (AICH group) and CG (Control group) showed AICH-induced pathological changes in the SHR rats. Also, 583 differentially expressed mRNAs were identified between MGT and MG, with 308 upregulated mRNAs and 275 downregulated mRNAs. Detailed information for genes is displayed in S9 Table. The 583 rat genes were converted into human genes based on homology mapping using Biomart package in R. Removing the duplicate values, 519 human genes were obtained. Compared with the putative 376 targets that
LXTYF for AICH, 14 genes were verified as follows: PTEN, CTH, PTGS2, PTAFR, FOS, NOS1, NOS2, PGR, MMP3, PON1, NEDD4, ACADSB, KL, and CASP3. The interaction network between 14 genes and other 403 genes was shown in Figure 6 at the help of the STRING database (cut off of confidence $=0.4$ ) and Cytoscape. Obviously, FOS, CASP3, and PTEN played an important role among them. Many important targets with expression changes, especially the 14 verified genes and the important gene nodes in the PPI network, are great markers of antioxidation, antiinflammatory, antiapoptosis, and lowering blood pressure, which indicated that LXTYF may play mutiroles in the mechanisms for AICH treatment.

3.7. KEGG Pathway Analysis of the mRNAs. In total, 519 differentially expressed mRNAs were submitted to KOBAS. The 33 enriched pathways that $p$ value $\leq 0.05$ were shown in Table 4. Expression changes of the genes and their related pathways of LXTYF for AICH treatment, such as the MAPK signaling pathway, calcium signaling pathway, Apoptosis, TNF signaling pathway, cGMP-PKG signaling pathway, cAMP signaling pathway, and HIF-1 signaling pathway, are involved in the biological processes of the antioxidation, 

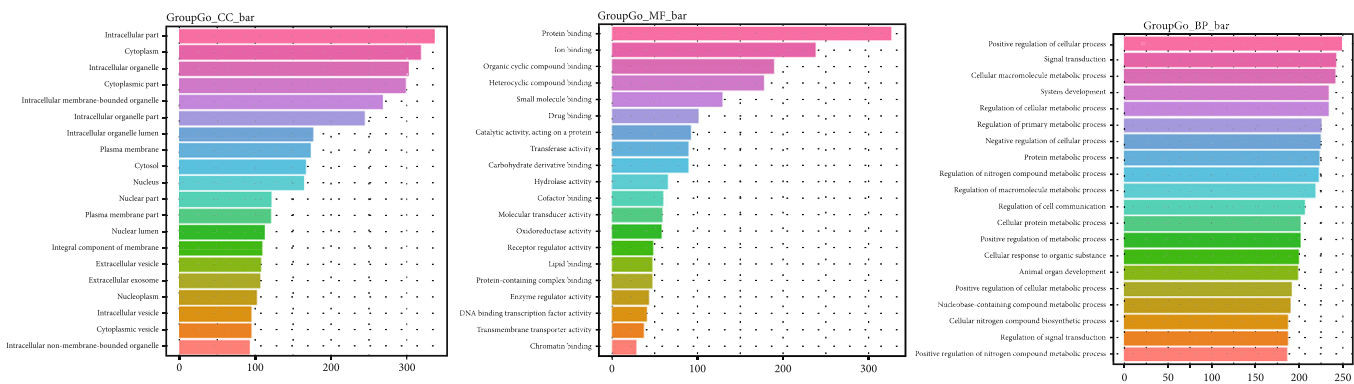

(a)

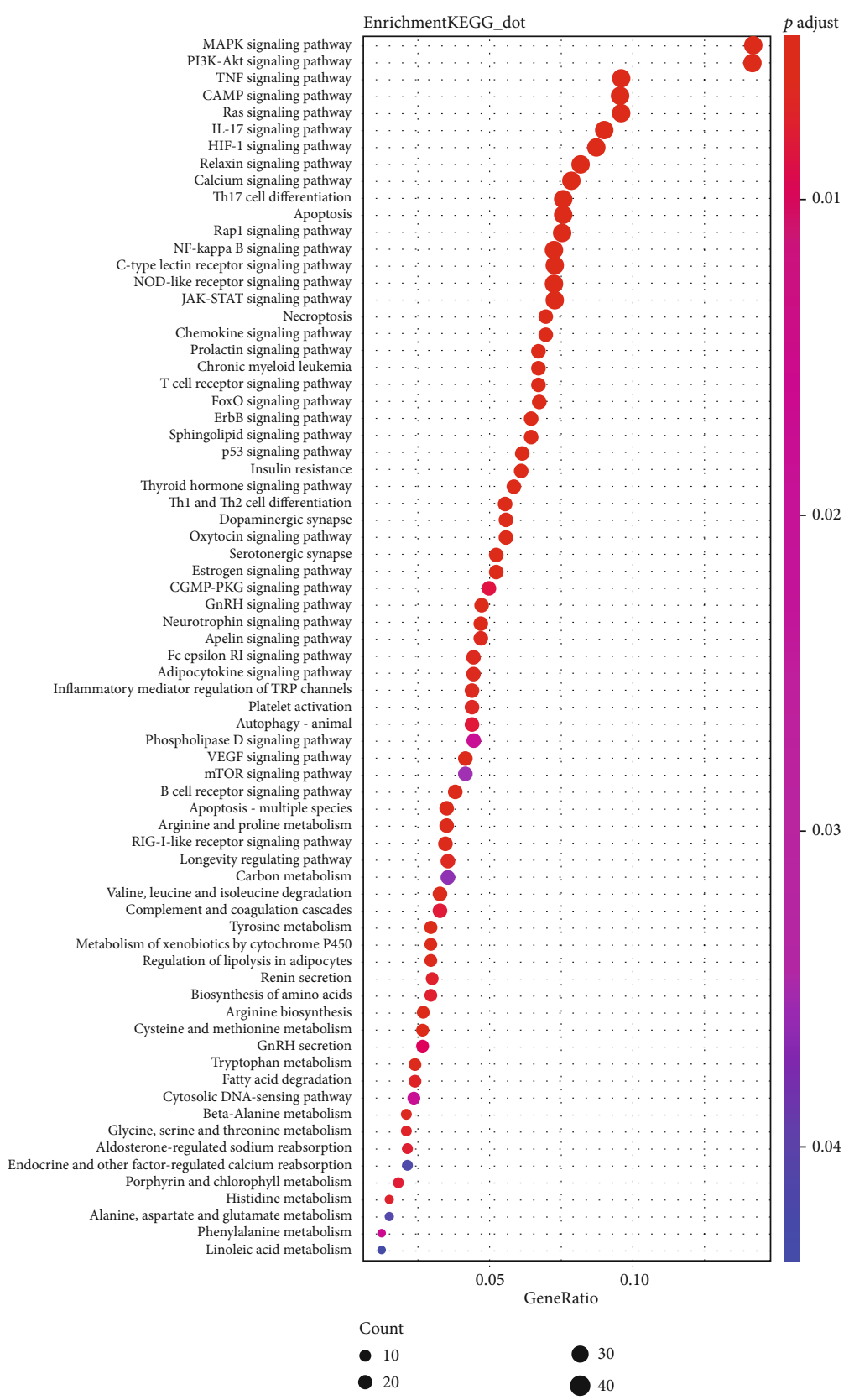

(b)

FIGURE 4: Enrichment analysis. (a) groupGO function for go ontology of 376 targets. The horizontal axis describes the number of genes and the vertical axis describes the GO terms. (b) enrichKEGG function for KEGG pathway enrichment analysis of 345 targets. The horizontal axis denotes generatio, and the vertical axis represents KEGG pathway terms. The size of the spot indicates the gene numbers enriched in each pathway and color indicates FDR value. 


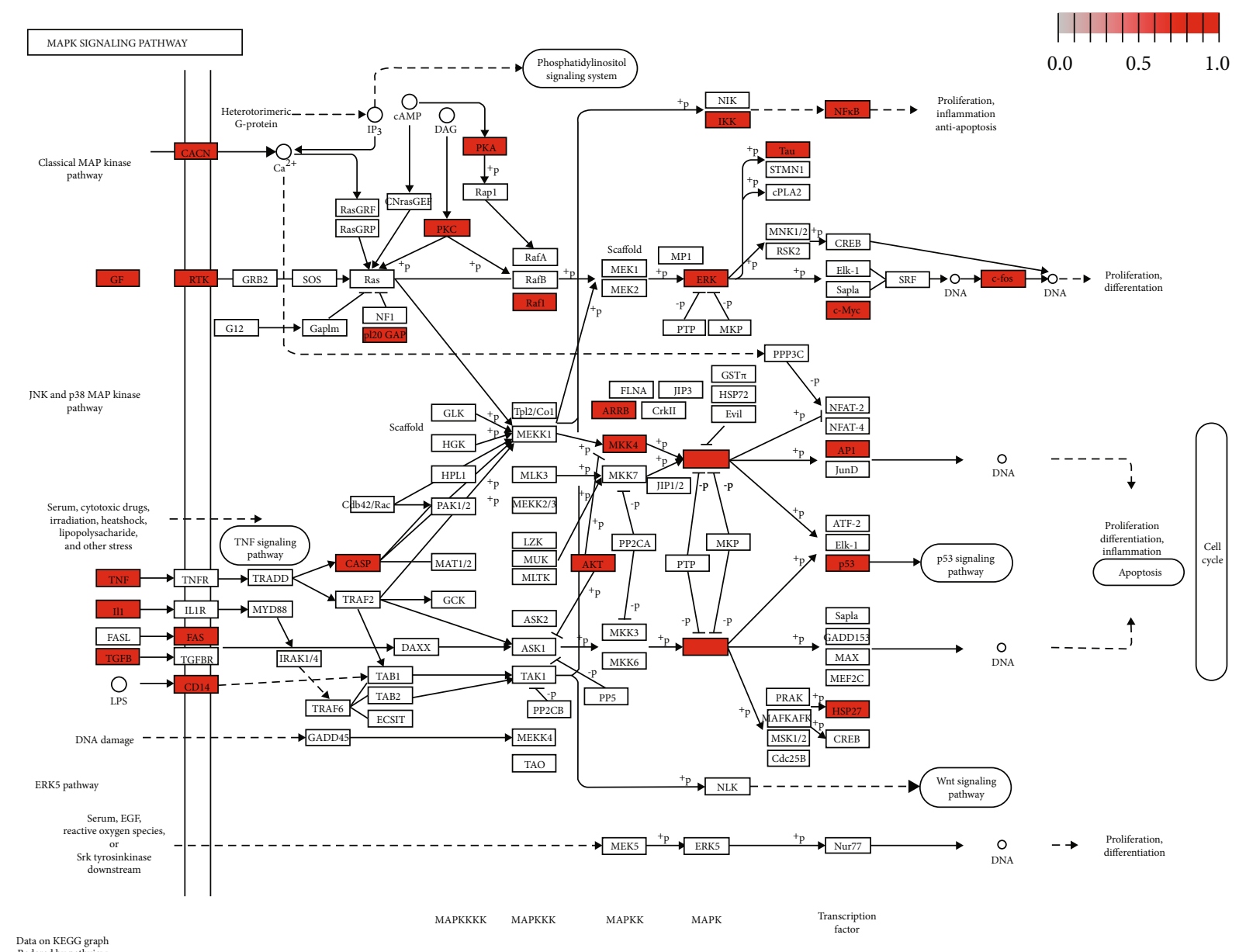

FIGURE 5: MAPK signaling pathways related to LXTYF for AICH. KEGG ID=hsa04010, enriched with 49 genes.

TABLE 3: Number of genes expressed in each group of RNA-seq data.

\begin{tabular}{lcccc}
\hline Group & Total genes & Differently expressed genes & Upregulated genes & Downregulated genes \\
\hline CGT vs. CG & 29049 & 613 & 324 & 289 \\
MG vs. CG & 29049 & 766 & 314 & 452 \\
MGT vs. MG & 29049 & 583 & 308 & 275 \\
\hline
\end{tabular}

CG: control group; CGT: the control group with LXTYF treatment; MG: ICH-model group; MGT: AICH model with LXTYF-treatment group.

anti-inflammatory, antiapoptosis, and lowering blood pressure.

Obviously, most pathways are metabolic pathways, and the analysis identified MAPK signaling pathway as the most correlated signaling pathway, which is consistent with the prediction of the former related pathways of LXTYF intervention on AICH. At the help of the KEGG mapper function in the KEGG database, 11 genes were enriched in the MAPK signaling pathway in Figure 7 as follows: GADD45G, DUSP1, FOS, NR4A11, IL1RAP, MEF2C, NLK, SRF, CASP3, CAC$\mathrm{NA} 1 \mathrm{H}$, and TAOK2.

3.8. Herb-Compound-Target-Pathway(H-C-T-P) Network Construction. In view of the mapping results between putative 72 pathways enriched by network pharmacology and 33 pathways and genes enriched in RNA-seq, 7 most common possible pathways were obtained. 38 differently expressed genes related to 7 pathways were achieved in RNA-seq data. Relevant 46 compounds and 8 herbs of the verified 14 genes were achieved from the data collected by network pharmacology. Network connections of the HerbCompound-Target-Pathway (H-C-T-P) verified in RNAseq were shown in Figure 8 and Table S10. In this network, nodes of degree among 10 33 which have great topological importance were as follows: CS and DaH in Herb, betasitosterol and quercetin in compound, PTAFR and CASP3 in Target, MAPK signaling pathway, and calcium signaling pathway in pathway. Details of connections among the 8 important nodes were shown in Table 5.

The results visually clarify the mechanisms of the multicomponent, multitarget, and multipathway of LXTYF on the treatment of AICH. Further studies can be made and 


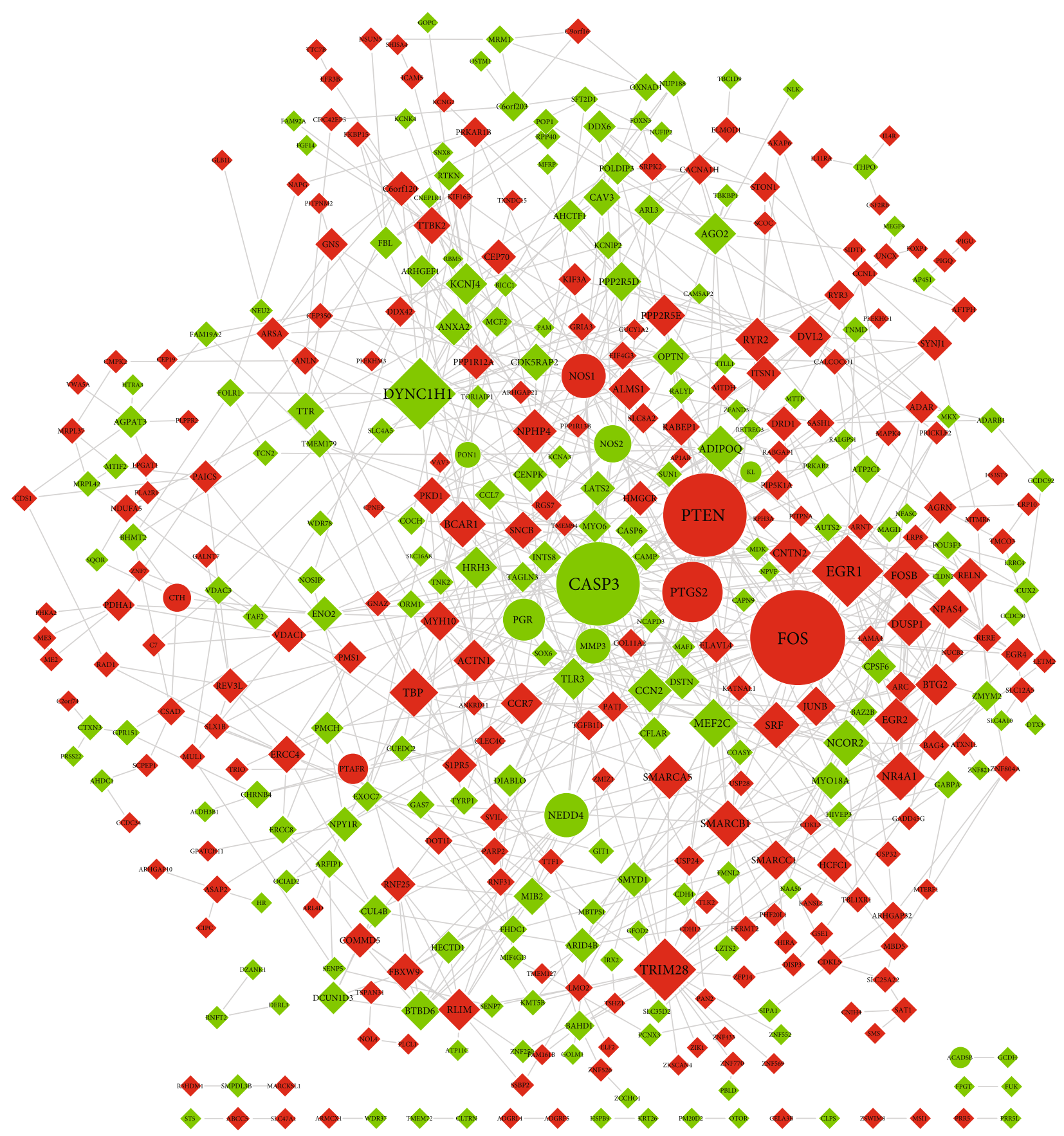

FIGURE 6: PPI network of identified genes and other differentially expressed genes from the RNA-seq. Compared with the MG, green shapes represent downregulated mRNAs, and red shapes represent upregulated mRNAs in MGT. Ellipses represent the verified genes treated by LXTYF for AICH, and diamonds represent other genes differentially expressed. A larger degree of the target nodes with larger shapes.

deeply investigate mechanisms upon the connections among the H-C-T-P network of LXTYF to treat AICH in our study.

\section{Discussion}

TCM has been developed and applied in China for more than 2000 years to prevent and treat diseases. To explore active ingredients, predict the targets, and reveal compounds action and drug-gene-disease associations in depth, TCM network pharmacology approach was constructed through "network target, multicomponents" mode [40]. The method is of predictable and systematic features. However, systematically investigating the scientific foundation of TCM formula for diseases at the molecular level is still a great challenge [40]. On the other hand, the development of modern molecular biology and genomic technologies, together with more advances in omics data and computational methods, can help to investigate complex biological pathways and mechanisms of medicines from molecular and cellular levels [41]. Li's previous study in our group showed LXTYF inhibited apoptosis 
TABLE 4: KOBAS pathway enrichment results of 583 differentially expressed mRNAs.

\begin{tabular}{|c|c|c|c|c|c|}
\hline Pathway name & ID & Input number & Background number & $p$ value & Corrected $p$ value \\
\hline Metabolic pathways & hsa01100 & 37 & 1243 & $4.87 E-06$ & 0.000776 \\
\hline MAPK signaling pathway & hsa04010 & 11 & 255 & 0.000719 & 0.022949 \\
\hline Calcium signaling pathway & hsa04020 & 11 & 180 & $4.07 E-05$ & 0.004326 \\
\hline Oxytocin signaling pathway & hsa04921 & 9 & 158 & 0.00033 & 0.014855 \\
\hline Regulation of actin cytoskeleton & hsa04810 & 9 & 215 & 0.002595 & 0.063668 \\
\hline Endocytosis & hsa04144 & 9 & 260 & 0.008413 & 0.107348 \\
\hline Apoptosis & hsa04210 & 8 & 140 & 0.000686 & 0.022949 \\
\hline Focal adhesion & hsa04510 & 8 & 203 & 0.006202 & 0.104136 \\
\hline Neuroactive ligand-receptor interaction & hsa04080 & 8 & 278 & 0.032413 & 0.198843 \\
\hline Circadian entrainment & hsa04713 & 7 & 95 & 0.000356 & 0.014855 \\
\hline TNF signaling pathway & hsa04668 & 7 & 110 & 0.000812 & 0.02355 \\
\hline cGMP-PKG signaling pathway & hsa04022 & 7 & 167 & 0.007476 & 0.106674 \\
\hline cAMP signaling pathway & hsa04024 & 7 & 199 & 0.017613 & 0.156542 \\
\hline Tight junction & hsa04530 & 6 & 139 & 0.011322 & 0.124199 \\
\hline RNA degradation & hsa03018 & 5 & 77 & 0.004147 & 0.094488 \\
\hline Phosphatidylinositol signaling system & hsa04070 & 5 & 98 & 0.010704 & 0.121948 \\
\hline Leukocyte transendothelial migration & hsa04670 & 5 & 118 & 0.02151 & 0.171545 \\
\hline AMPK signaling pathway & hsa04152 & 5 & 125 & 0.026528 & 0.188053 \\
\hline Dopaminergic synapse & hsa04728 & 5 & 130 & 0.030533 & 0.1948 \\
\hline Cell adhesion molecules (CAMs) & hsa04514 & 5 & 146 & 0.045802 & 0.231919 \\
\hline Arginine and proline metabolism & hsa00330 & 4 & 50 & 0.005113 & 0.10411 \\
\hline Lysine degradation & hsa00310 & 4 & 52 & 0.005821 & 0.10411 \\
\hline Inositol phosphate metabolism & hsa00562 & 4 & 71 & 0.015938 & 0.154071 \\
\hline mRNA surveillance pathway & hsa03015 & 4 & 92 & 0.035408 & 0.200721 \\
\hline Fc gamma R-mediated phagocytosis & hsa04666 & 4 & 93 & 0.036571 & 0.201138 \\
\hline HIF-1 signaling pathway & hsa04066 & 4 & 103 & 0.049393 & 0.242404 \\
\hline Pyruvate metabolism & hsa00620 & 3 & 40 & 0.017749 & 0.156542 \\
\hline Cysteine and methionine metabolism & hsa00270 & 3 & 45 & 0.023739 & 0.180305 \\
\hline Nucleotide excision repair & hsa03420 & 3 & 47 & 0.026404 & 0.188053 \\
\hline Notch signaling pathway & hsa04330 & 3 & 48 & 0.027794 & 0.18808 \\
\hline Fanconi anemia pathway & hsa03460 & 3 & 55 & 0.038589 & 0.206572 \\
\hline Arginine biosynthesis & hsa00220 & 2 & 21 & 0.034827 & 0.200721 \\
\hline Glycosylphosphatidylinositol(GPI)-anchor biosynthesis & hsa00563 & 2 & 25 & 0.046729 & 0.232917 \\
\hline
\end{tabular}

and promoted proliferation in PC12 cells damaged by Lglutamate. Also, a decrease in levels of functional proteins in SHRs such as TNF-a, IL-1, D2D, S-100B, and NSE after LXTYF administration showed LXTYF exerted great pharmacology effect related to anti-inflammatory, anticoagulation, and blood vessel protection activity [14]. Huang's study also showed the cooling-blood and activating-blood effects of LXTYF and its parts via decreases in rectal temperature, blood viscosity, and plasma viscosity of SD rats [42]. In a rat model induced by intrastriatal autologous blood injection, LXTYF significantly ameliorated brain edema after AICH by upregulating metalloproteinase-1 (TIMP-1) and inhibiting metalloproteinase-9 (MMP-9) expression [11].

Network pharmacology and transcriptomics data were applied in this study to understand the underlying different mechanisms and actions of LXTYF for AICH treatment. We successfully identified 76 active ingredients (quercetin, alanine, kaempferol, and so on) of LXTYF and 376 putative targets to alleviate AICH (PTGS2, PTGS1, ESR1, and so on). PPI network indicated the important role of STAT3 in the proteins-proteins interaction. Gene Ontology and KEGG pathway enrichment analysis indicated that LXTYF is most likely to influence the MAPK signaling pathway and PI3KAkt signaling pathway. From the RNA-seq data of AICHrats, 583 differentially expressed mRNAs were identified, and 14 of them were consistent with the putative targets of LXTYF treatment of AICH. KEGG pathway enrichment results showed that the MAPK signaling pathway was the most correlated one among the pathways.

4.1. Ingredients and Targets of LXTYF for AICH. Ingredienttarget-pathway analysis on herb prescriptions is helpful to uncover the complicated mechanisms underlying diseases and the therapy. Network pharmacology is an effective method 


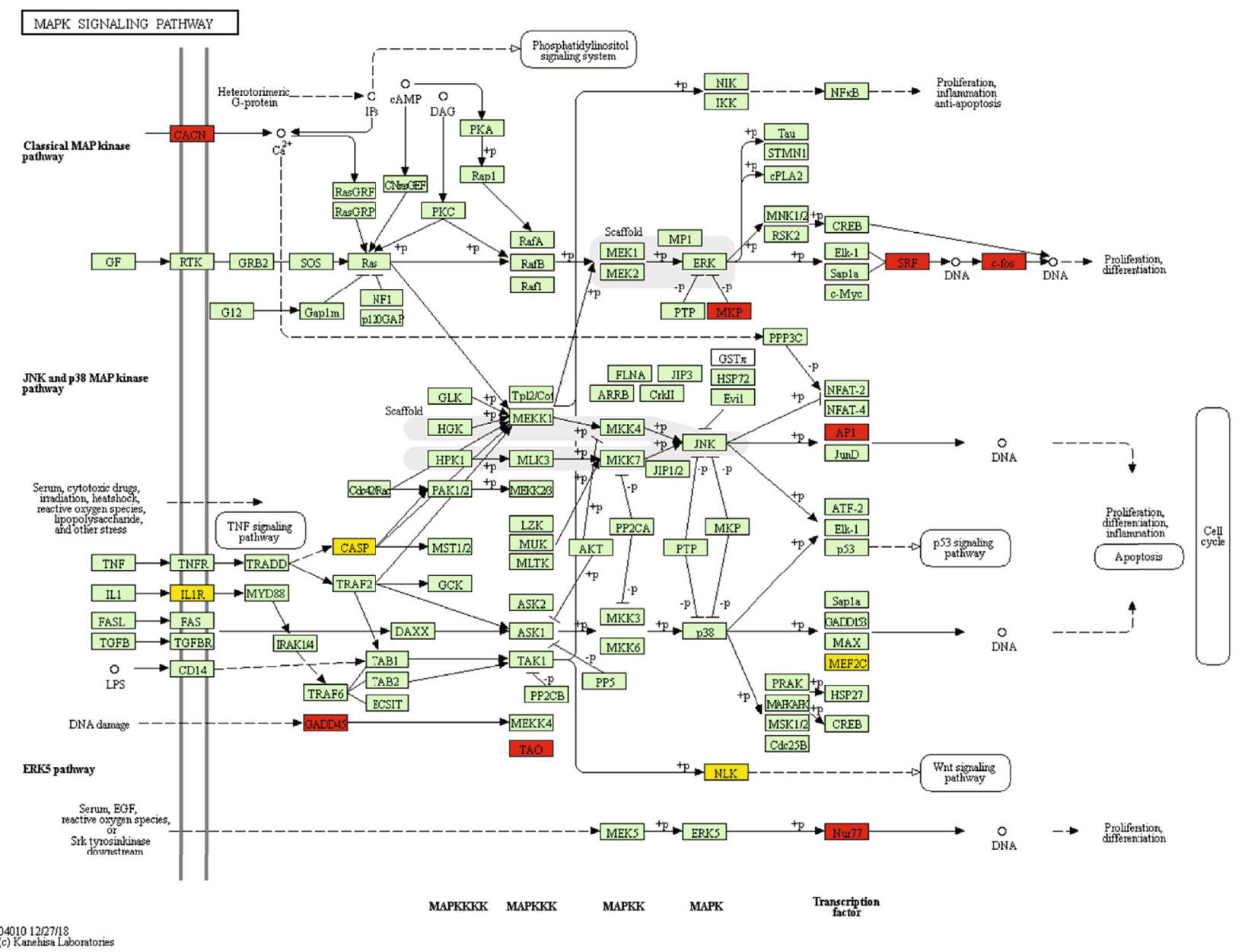

FIGURE 7: The most correlated MAPK signaling pathway enriched in KEGG mapper. All green boxes represent targets of MAPK signaling pathway in human. 8 red boxes (GADD45(GADD45G), MKP (DUSP1), c-fos (FOS), AP1 (FOS), Nur77(NR4A11), SRF, CACN (CACNA1H), and TAO(TAOK2)) represent upregulated targets. 4 yellow boxes (IL1R(IL1RAP), MEF2C, NLK, and CASP(CASP3)) represent downregulated targets.

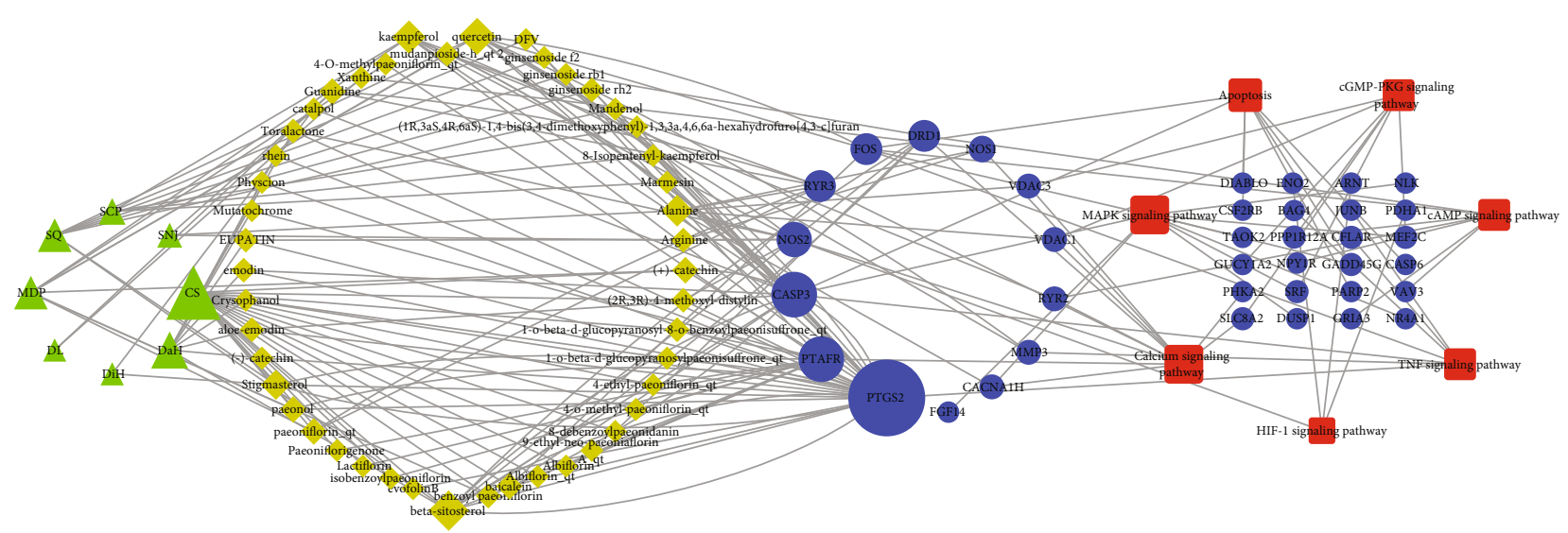

FIgure 8: Herb-Compound-Target-Pathway(H-C-T-P) verified network related to the treatment of LXTYF for AICH in RNA-seq. Green triangles represent 8 herbs, yellow diamonds represent 46 compounds, blue ellipses represent 38 targets, and red round rectangles represent 7 pathways. A larger degree of the target nodes with larger shapes.

to deeply investigate the mechanisms of TCMs to treat $\mathrm{AICH}$ through muti-ingredients and mutitargets. In this study, 76 active ingredients and 376 targets of LXTYF for treating AICH were obtained at the help of $2315 \mathrm{ICH}$ targets, the deeper results than the 34 active ingredients, 146 targets, and the $1436 \mathrm{ICH}$ targets in our former study [14]. 
TABLE 5: Details of connections of important nodes in the H-C-T-P network.

\begin{tabular}{|c|c|c|}
\hline Connections & Node 1 & Node 2 \\
\hline Herb-Cpmpound & CS & $\begin{array}{l}\text { (+)-Catechin, (2R,3R)-4-methoxyl-distylin, 1-o-beta-d- } \\
\text { glucopyranosyl-8-o-benzoylpaeonisuffrone_qt, 1-o-beta-d- } \\
\text { glucopyranosylpaeonisuffrone_qt, baicalein, } \\
\text { benzoylpaeoniflorin, beta-sitosterol, evofolin B, } \\
\text { isobenzoylpaeoniflorin, lactiflorin, paeoniflorigenone, } \\
\text { paeoniflorin_qt, paeonol, 4-ethyl-paeoniflorin_qt, 4-o- } \\
\text { methyl-paeoniflorin_qt, 8-debenzoylpaeonidanin, 9-ethyl- } \\
\text { neo-paeoniaflorinA_qt, albiflorin, albiflorin_qt, } \\
\text { stigmasterol }\end{array}$ \\
\hline Herb-Cpmpound & $\mathrm{DaH}$ & $\begin{array}{l}\text { (-)-Catechin, aloe-emodin, beta-sitosterol, crysophanol, } \\
\text { emodin, EUPATIN, mutatochrome, physcion, rhein, } \\
\text { toralactone }\end{array}$ \\
\hline Compound-target & $\begin{array}{l}\text { 1-O-beta-d-glucopyranosyl-8-o-benzoylpaeonisuffrone_- } \\
\text { qt, 1-o-beta-d-glucopyranosylpaeonisuffrone_qt, 4-ethyl- } \\
\text { paeoniflorin_qt, 4-O-methylpaeoniflorin_qt, 4-o-methyl- } \\
\text { paeoniflorin_qt, 9-ethyl-neo-paeoniaflorinA_qt, } \\
\text { albiflorin, albiflorin_qt, benzoylpaeoniflorin, } \\
\text { ginsenosidef2, isobenzoylpaeoniflorin, lactiflorin, } \\
\text { mutatochrome, paeoniflorigenone }\end{array}$ & PTAFR \\
\hline Compound-target & $\begin{array}{l}\text { Aloe-emodin, baicalein, beta-sitosterol, catalpol, emodin, } \\
\text { ginsenosiderh2, kaempferol, quercetin }\end{array}$ & CASP3 \\
\hline Target-pathway & $\begin{array}{l}\text { CACNA1H, CASP3, DUSP1, FGF14, FOS, GADD45G, } \\
\text { MEF2C, NLK, NR4A1, SRF, TAOK2 }\end{array}$ & MAPK signaling pathway \\
\hline Target-pathway & $\begin{array}{l}\text { CACNA1H, PTAFR, DRD1, NOS1, NOS2, PHKA2, } \\
\text { RYR2, RYR3, SLC8A2, VDAC1, VDAC3 }\end{array}$ & Calcium signaling pathway \\
\hline
\end{tabular}

The active ingredients involved in eight herbs were screened by $\mathrm{OB}$ and DL values, which represent the vast majority of the ingredients absorbed into the blood circulation and pharmaceutical properties of compounds in the prescription, and form a relatively complete chemical component library. To some extent, they can reflect the pharmacodynamic ingredients of LXTYF that may play a role in the clinical medication. Results of the active component-target network of LXTYF to treat AICH showed that most components affected multiple targets. Quercetin, alanine, and kaempferol from MDP/SQ, SNJ, and MDP/SCP acted on 112,104 , and 39 targets, respectively. They are of high OB and DL values and regulated most targets for AICH treatment, also with anti-inflammatory, antioxidant, and antiapoptotic properties. The findings were consistent with previous studies as follows. Alanine was reported to have antioxidant and procoagulant efficacy [10, 43]. D-alanine D-leucine enkephalin (DADLE) has anti-inflammatory, antioxidant, neuroregenerative, and antiapoptotic properties [44]. Quercetin can promote neuronal and behavioral recovery by its antioxidant activity and restraining inflammatory response and apoptosis in a rat model of $\mathrm{ICH}[45,46]$. Kaempferol can effectively prevent nitrosative-oxidative stress after ischemia/reperfusion via the mechanism of inhibiting the nitrotyrosines and preventing apoptosis by degrading caspase- 9 activity and poly-(ADP-ribose) polymerase in model rats [47]. On the other hand, kaempferol glycosides suppress brain injury and inflammation by inhibiting the activation of NF- $\kappa$ B and STAT3 in stroke rats [48]. So, quer- cetin, alanine, and kaempferol might reasonably be regarded as crucial active compounds of LXTYF to treat AICH.

Numerous constituents make up the complexed TCM system, and combinations of them play a role in the treatment of diseases. From the H-C-T-P network, the compounds of animal herbs like xanthine and guanidine in DL and guanidine, arginine and alanine in SNJ play vital role in the putative mechanisms of LXTYF to treat AICH. SNJ has been a good substitute for Rhinoceri Asiatici Cornu (a highly endangered species) since the 1970s because it is abundant, has low price and with similar inorganic, amino acid constituents, and comparable pharmacological properties [10]. Thus, it is favorable to keep the balance between legislation and apparent indispensability of Rhinoceri Asiatici Cornu in TCM. The research and discovery of the active ingredients in this study will contribute to find alternative medicines of endangered animals to better protect animals and treat diseases.

Via evaluating topological paramters, among all the putative targets of LXTYF associated with AICH, PTGS2, Bax, TNF, JUN, IL1B, and NOS2 are examples of relatively important targets which are involved in MAPK, PI3K-Akt, TNF, and apoptosis signaling pathways, while RNA-seq analysis showed that the LXTYF administration significantly regulated 583 gene transcriptions in $\mathrm{AICH}$ rat models. Combined with putative targets in network pharmacology, a total of 14 genes were verified. PTEN, CTH, PTGS2, PTAFR, FOS, and NOS1 are six upregulated genes, and NOS2, PGR, MMP3, PON1, NEDD4, ACADSB, KL, and 
CASP3 are eight downregulated genes. Especially CTH, PTAFR, PGR, PON1, NEDD4, ACADSB, and KL are 7 new genes compared with our previous study [49]. And PPI network of them showed that FOS, PTEN, and CASP3 were core genes playing important roles among the differently expressed genes.

PTGS2 and PTGS1 (Prostaglandin G/H synthase, PTGS) are genes that code for the COX-2 and COX1 (COX enzymes, cyclooxygenase) proteins, respectively [50]. COX enzyme is a key therapeutic target, and its inhibitors such as NSAIDs are widely used in clinical treatment for inflammation, pain, fever, and son on [50]. But inhibition of COX-2 conveys a definite risk of myocardial infraction and stroke due to thrombosis and hypertensive effects [51]. Therefore, upregulation of the PTGS2 gene indicates that LXTYF may reduce the risk of blood pressure elevation, which is conducive to antihypertensive therapy in the early stage of an intracerebral hemorrhage.

Phosphatase and tensin homolog deleted on chromosome 10 (PTEN) is a tumor suppressor, a proapoptotic gene mainly via inactivation of PI3K/AKT signaling pathway, and PTEN conditional knockouts have become a potentially approach targeting cancer and obesity treatment [52]. But in stroke treatment, PTEN is a double-edged sword [53]. The previous study showed that PTEN was upregulated in AICH stage, and its inhibition has been reported to be neuroprotective against ischemic stroke in experimental models $[53,54]$. While it has been demonstrated that PTEN deletion can lead to cognitive impairment and PTEN loss in neurons and astrocytes is, respectively, unfavorable to long-term functional recovery, exacerbates damage, enhances astrogliosis, and induces inflammation [53]. So, upregulation of PTEN in our study may show that LXTYF treatment has no harm on cognition function and play roles on neuronaldamage recovery after $\mathrm{AICH}$.

Nitric oxide synthases (NOS), with 3 isoforms called neuronal NOS (nNOS/NOS1), inducible NOS (iNOS/NOS2), and endothelial NOS (eNOS/NOS3), form NO and play dual roles of damage and protection in $\mathrm{ICH}$ [55]. NO formed via NOS1 and NOS3 gives rises to vasodilatation, hypotension, inhibitions of platelet aggregation, and adhesion, and as an antioxidant, it exerts other beneficial actions. NOS1 has great significance in controlling salt-water balance, and blood pressure, especially the long-term blockade of NOS1 by 7-NI, a specific inhibitor of NOS, leads to hypertension in rats [55], while NO through NOS2 during inflammation increases oxidative and nitrative stress, causing neurodegeneration and enhancement of apoptosis [56]. Selective NOS2 inhibitors that exhibit neuroprotective properties can be candidate treatments for acute ischemic stroke [57]. So, LXTYF may play function of vasodilatation and antihypertension via upregulation of NOS1 and supress oxidative stress and apoptosis via downregulation of NOS2 to treat $\mathrm{AICH}$.

FOS is one of the components of AP1, the inducer of cell proliferation. It can activate nerve growth factor and has gene repair activities after neuronal death in the stroke model. Knockdown of c-fos enhances necrosis significantly [58]. But serving as an immediate-early gene, its role of expression is complex and has neurotoxic and neuroprotective effects. Especially its neuroprotective effect is mainly on gene repair activities after neuronal death, with intact c-fos transcript playing roles in the necrosis core and oxidative DNA lesions [58]. So, upregulated changes of FOS in RNA-seq showed that LXTYF may have neuroprotective effect and attenuated oxidative DNA lesions and necrosis after $\mathrm{AICH}$, similar to 7-nitroindazole (7-NI). 7-NI is a specific inhibitor of NOS and can increasec-fos mRNA levels and attenuate oxidative DNA lesions, nitric oxide, and necrosis after stroke [58]. But several previous reports showed that upregulation of cFos after AICH leads to neuronal apoptosis, and FOS is regarded to be neurotoxic. So, role of FOS in AICH also needs further study.

Caspase 3, a member of caspases family involved in cell death, has been recognized as a crucial mediator of neuronal apoptosis [59]. Stroke is usually of great neuronal cell death and serum caspase- 3 concentrations rise in rat models and AICH patients [59-61]. Downregulation of Caspase 3 after administration in this study shows that LXTYF may treat $\mathrm{AICH}$ via apoptosis inhibition.

4.2. Pathway Analysis of LXTYF for AICH Treatment. Multicomponent, multitarget, and multipathway are general features of TCMs, and LXTYF has therapeutic effects by making contributions to several pathways. In this present work, putative targets were enriched significantly in 72 KEGG pathways, and the two most important enriched pathways were the MAPK signaling pathway and PI3K-Akt signaling pathway. Our previous study has verified that LXTYF inhibited the apoptosis to treat AICH based on the PI3K-AKT pathway on the level of transcription and protein [14]. Meanwhile, MAPK (mitogen-activated protein kinase) signaling pathway is the most important enriched and verified pathway in our RNA-seq analysis, which plays roles in modulating oxidative stress, inflammatory action, and cell death [62]. It can response to extracellular stimuli, then sequential phosphorylation of kinase cascades MAPKK kinases (MAPKKKs), MAPK kinases (MAPKKs), dual phosphorylation of the tripeptide motif (Thr-X-Tyr), and MAPK were activated, thus transmitting signals to the nucleus [63]. MAPK family consists of four subfamilies: extracellular signal-regulated kinase 1/2 (ERK1/2), p38 kinase, c-Jun amino terminal kinase (JNK), and ERK5. It has been viewed as a potential therapeutic target for stroke and hemorrhagic cerebral vascular disease [64]. Studies have found that the balance of MAPK activities determines cell fate. ERK is activated by various growth factors and shows benefits to cell growth and cellular survival. In contrast, p38 and JNK are activated by stress conditions, leading to inflammation and apoptosis via various mechanisms [65]. Some preclinical researches proved that MAPK inhibition (MAPKi) can dramatically increase the efficacy of immunotherapy, especially in cancer types [66]. Proinflammatory cytokines (interleukin-1beta (IL-1 $\beta$ ), tumor necrosis factor-alpha (TNF- $\alpha$ )) and transforming growth factor-beta (TGF- $\beta$ ) activate p38 MAPK, and its specific inhibitors were used as the treatment of peripheral inflammatory diseases [64]. It is known that p38s and ERK5 can trigger phosphorylation of MEF2c and 
then increase c-Jun expression [67, 68], which is distinct among several diseases like brain ischemia, injury, cell death, neuro-inflammatory, and neurodegenerative disease [69]. MEF2C, a member of the myocyte-enhancer factor 2 (MEF2) group of transcription factors, was verfied to be downregulated. Thus, it is reasonable to speculate that p38 MAPK was inhibited; phosphorylation of MEF2C and c-jun mRNA transcription was decreased to prevent inflammatory response and apoptosis for treatment of AICH after LXTYF administration [70, 71]. Proteins of the Fos and Jun families can form homodimers or heterodimers called AP-1 (activating protein-1) to mediate transcriptional activation and DNA binding activity on target genes, involved in apoptotic functions and cell proliferation [66, 72]. Most time c-Fos is a necessary inducer of cell proliferation during the cell cycle phases in growing cells [67]. FOS and FOSB, verified to upregulated, are possible to play the proliferative role and induce compensatory cell proliferation via JNK and ERK pathways for the treatment of AICH after LXTYF administration. Dual-specificity protein phosphatase (DUSP) is an antiapoptotic phosphatase, which can dephosphorylate and inactivates the downstream effectors such as JNK, p38, and ERK $[65,73]$. Downregulation of DUSP1 and increase of JNK phosphorylation were observed in the IR injury of the heart. Reproduction of DUSP1 can not only reduce the cellular death and shows the protective effects but also limit inflammatory responses upon LPS stimulation $[65,73,74]$. So, upregulation of DUSP1 shows that LXTYF may have a decrease in MAPKs phosphorylation and inactivates JNK and p38 pathways, suppressing inflammation and apoptosis to treat $\mathrm{AICH}$.

Moreover, the other pathways such as the calcium signaling pathway, apoptosis, TNF signaling pathway, cGMP-PKG signaling pathway, cAMP signaling pathway, and HIF-1 signaling pathway are the common signaling pathways of differentially expressed genes and putative targets in LXTYF. The calcium signaling pathway generates $\mathrm{Ca}^{2+}$ signals to regulate physiological processes [75]. Research progress shows that low blood calcium leads to increased hematoma volume and poor prognosis of $\mathrm{AICH}$, and calcium intake in the diet can lower the risk of stroke by about $24 \%$ [76]. Blood calcium on reducing stroke is mainly from several mechanisms such as inhibition of fat synthesis, lowering of blood pressure, and stability of blood glucose [76]. Activation of the apoptosis pathway is known to contribute to neuronal loss and become the core problem in the pathogenesis of stroke [77]. Bcl-2 family proteins and caspase proteins regulate neuronal apoptosis via mitochondria and ER. What is more, oxidative and nitrosative stress after stroke injury will lead to apoptotic cell death [77]. DIABLO (direct IAPbinding protein with low $\mathrm{pI}$ ) is released from mitochondria into the cytosol, interacts with multiple IAPs, and removes IAP-mediated inhibition of caspases [78]. So, when it comes to the downregulation of DIABLO, Caspase-3, and Caspase- 6 in RNA-seq data, it is reasonable to speculate the mechanism that LXTYF can help to decrease mRNA expression of DIABLO, then reduced inhibition of IAPs and promoted inhibition of caspase-3 and caspase-6. The TNF signaling pathway plays pleiotropic roles in the CNS [79]. Tumor necrosis factors (TNF) consists of soluble TNF (solTNF) and transmembrane TNF (tmTNF), which has a preference for TNF receptor 1 (TNFR1) and TNF receptor 2 (TNFR2), respectively [79]. Observations have suggested that in acute stroke and reduce injury, solTNF action can be inhibited by intraventricular infusion of the TNFR1 decoy receptor, but in the aspect of hippocampal repair and neurogenesis after ischemic injury, tmTNF action through TNFR2 is crucial [79]. Therefore, the adverse effects of anti-TNF therapies in the CNS may be reduced by selectively supressing TNFR1mediated signaling while sparing TNFR2 activation [79]. BAG4/SODD is an antiapoptotic protein and identified as a silencer of death domain that can bind to TNFR1. High levels of SODD/BAG-4 protect against TNFa-induced cell death. So, elevation of BAG-4 indicates the control of LXTYF on the TNF signaling pathway.

4.3. Limitations of the Research. Compared to the previous two papers published in Frontiers in Pharmacology and Journal of Ethnopharmacology, all studies used reasonable models and reliable validation data to detect the network effect of LXTYF to treat AICH and verified some components, targets, and pathways [14, 78]. But our current work is different in the following aspects. We think that comprehensive ingredients in the prescription, the method with low false positive for pathway enrichment, and detailed differential mRNA expression can more systematically and efficiently validate the network pharmacology effects. Results in our study can supplement the mechanisms of LXTYF on AICH in a more systematic and comprehensive way. We collected compounds of all 8 herbs; more ICH targets and PPI network were analyzed in detail. A more structured and detailed network pharmacology approach via packages in $\mathrm{R}$ was also applied in our study. Also, compared with the predictive network and RNA-seq analysis results, new genes and new pathways (14 genes and MAPK signaling pathway) were verified and enriched. In the future, more targets and pathways will be explored deeply, conformed, and fully depict the pharmacology mechanism of TCM on AICH.

There are still some limitations to our study. The insufficient research on medicine composition of this prescription, especially the animal herbs, cannot completely dig into all the effective components. Further progress of chemical component separation technology is in great need to fully clarify all chemical components in the prescription. Also, results of PPI network prediction showed that STAT3 may play an important role of the bridge to connect other proteins among the pharmacological actions, but it did not verify in RNA-seq and needs to investigate in the next future. More subsequent experiments on differently expressed mRNA and enriched pathways are necessary to be investigated deeply. Moreover, it is common to see that some genes have dual effects and play opposite roles in different physical conditions, especially some are unsure and need further study. Critical insight for targets and pathways are necessary for mutual promotion between pharmacological research and physiological/pathology research. 


\section{Conclusion}

Taken together, network pharmacology method and enrichment analysis in $\mathrm{R}$ were applied in our study to identify the 76 active components and 376 putative targets of LXTYF alleviating AICH. Compared with RNA-seq results of AICH rat models and enrichment analysis, we found that LXTYF attenuates AICH partially by regulating the targets involved MAPK, calcium, apoptosis, and TNF signaling pathway and plays antioxidation, anti-inflammatory, antiapoptosis and lowers blood pressure roles in the treatment mechanisms.

\section{Data Availability}

The chemical ingredients of six botanical medicinal materials in LXTYF were obtained from Traditional Chinese Medicine Systems Pharmacology Database and Analysis Platform (TCMSP) (http://lsp.nwu.edu.cn/tcmsp.php) The chemical ingredients of the other two animal medicines in LXTYF were derived from BATMAN-TCM (http://bionet .ncpsb.org/batman-tcm). Different genes linked to ICH were collected from three existing resources with Medical Subject Heading (MeSH) "Cerebral Hemorrhage": (1) GAD (https:// geneticassociationdb.nih.gov/), (2) Genecards (https://www .genecards.org/), and (3) DisGeNET (http://www.disgenet .org/web/DisGeNET).

\section{Conflicts of Interest}

The authors declare that there is no conflict of interest regarding the publication of this article.

\section{Authors' Contributions}

YC designed the study, performed the network pharmacological analysis and RNA-seq data analysis, and wrote the manuscript. JD analyzed the data and reviewed the manuscript. DY performed the quality control of statistical analysis. QQ, PW, $\mathrm{XY}$, and WL performed the animal experiments. GL also designed the study, with XS and FW revised the manuscript as corresponding authors. All authors read and approved the final manuscript. Yang Chen and Ju Dong are first authors.

\section{Acknowledgments}

This work was supported by three grants from the Natural Science Foundation of Jiangsu Province of China (Grant no.: BK20161575), National Natural Science Foundation of China (Grant no.: 81373512), and the Priority Academic Program Development of Jiangsu Higher Education Institutions (integration of Chinese and western medicine). Funding body provided the funds, and funds were used to afford the cost of the rats and RNA sequencing of samples in companies.

\section{Supplementary Materials}

S1 Table. 517 compounds of 8 herbs in LXTYF collected from TCMSP and BATMAN-TCM database. S2 Table. 874 targets of the active ingredients obtained from the TCMSP. S3 Table.
149 targets of active components predicted from the SwissTargetPrediction database. S4 Table. 2315 targets relating to ICH collected from GAD, Genecards, and DisGeNET database. S5 Table. Topological parameters of component-target network. S6 Table. Proteins-proteins interaction information of LXTYF targets for AICH in STRING 11.0. S7 Table. Topological parameters of the PPI network. S8 Table. Results of putative targets for KEGG pathway enrichment analysis. S9 Table. Detailed information of 583 differentially expressed mRNAs identified between MGT and MG. S10 Table. Details of the Herb-Compound-Target-Pathway(H-C-T-P) network connection verified in RNA-seq. (Supplementary Materials)

\section{References}

[1] R. Rodrigo, R. Fernandez-Gajardo, R. Gutierrez et al., "Oxidative stress and pathophysiology of ischemic stroke: novel therapeutic opportunities," CNS \& Neurological Disorders Drug Targets, vol. 12, no. 5, pp. 698-714, 2013.

[2] T. Emiru, E. M. Bershad, N. D. Zantek et al., "Intracerebral hemorrhage," Clinical and Applied Thrombosis/Hemostasis, vol. 19, no. 6, pp. 652-662, 2013.

[3] A. Fahlström, L. Tobieson, H. N. Redebrandt et al., "Differences in neurosurgical treatment of intracerebral haemorrhage: a nation-wide observational study of 578 consecutive patients," Acta Neurochirurgica, vol. 161, no. 5, pp. 955-965, 2019.

[4] A. I. Qureshi, S. Tuhrim, J. P. Broderick, H. H. Batjer, H. Hondo, and D. F. Hanley, "Spontaneous intracerebral hemorrhage," The New England Journal of Medicine, vol. 344, no. 19, pp. 1450-1460, 2001.

[5] C. Cordonnier, A. Demchuk, W. Ziai, and C. S. Anderson, "Intracerebral haemorrhage: current approaches to acute management," Lancet, vol. 392, no. 10154, pp. 1257-1268, 2018.

[6] R. F. Keep, Y. Hua, and G. Xi, "Intracerebral haemorrhage: mechanisms of injury and therapeutic targets," Lancet Neurology, vol. 11, no. 8, pp. 720-731, 2012.

[7] J. C. Chen, "The effects of acupuncture and traditional Chinese medicines on apoptosis of brain tissue in a rat intracerebral hemorrhage model," Physiology \& Behavior, vol. 151, pp. 421-425, 2015.

[8] Y. Wang, Y. C. Fan, C. L. Xie, and G. Q. Zheng, "History of post-stroke epilepsy in ancient China," Journal of Neurology, vol. 258, no. 8, pp. 1555-1558, 2011.

[9] H. Q. Li, J. J. Wei, W. Xia et al., "Promoting blood circulation for removing blood stasis therapy for acute intracerebral hemorrhage: a systematic review and meta-analysis," Acta Pharmacologica Sinica, vol. 36, no. 6, pp. 659-675, 2015.

[10] R. Liu, Q. Huang, J. Shan et al., "Metabolomics of the antipyretic effects of Bubali Cornu (water buffalo horn) in rats," PLoS One, vol. 11, no. 7, article e0158478, 2016.

[11] C. Y. He, J. H. Huang, W. J. Wang et al., "Effects of Liangxue Tongyu Formula on brain edema and expressions of matrix metalloproteinase-9 and tissue inhibitor of metalloproteinase-1 in rats with intracerebral hemorrhage," Journal of Chinese Integrative Medicine, vol. 8, no. 4, pp. 347-351, 2010.

[12] W. F. Guo, L. K. Zhang, M. H. Wu et al., "Liangxue Tongyu Fang for treating acuter-phase cerebral hemorrhage in 168 cases," Journal of Beijing University of Traditional Chinese Medicine, vol. 35, no. 9, pp. 603-616, 2012. 
[13] M. A. Hui-Min, W. F. Guo, Y. Yuan, H. X. Zhang, and L. I. Jian-Xiang, "Treatment with Liangxuetongyu Formula in 96 patients with syndrome of stasis-heat obstructing orifices of acute cerebral hemorrhage," Journal of Beijing University of Traditional Chinese Medicine, vol. 34, no. 5, pp. 348-352, 2011.

[14] X. Li, X. Huang, Y. Tang et al., "Assessing the pharmacological and therapeutic efficacy of traditional Chinese medicine Liangxue Tongyu prescription for intracerebral hemorrhagic stroke in neurological disease models," Frontiers in Pharmacology, vol. 9, p. 1169, 2018.

[15] G. Bai, Y. Y. Hou, M. Jiang, and J. Gao, "integrated systems biology and chemical biology approach to exploring mechanisms of traditional Chinese medicines," Chinese Herbal Medicines, vol. 8, no. 2, pp. 99-106, 2016.

[16] J. Ru, P. Li, J. Wang et al., "TCMSP: a database of systems pharmacology for drug discovery from herbal medicines," Journal of Cheminformatics, vol. 6, no. 1, p. 13, 2014.

[17] Z. Liu, F. Guo, Y. Wang et al., "BATMAN-TCM: a bioinformatics analysis tool for molecular mechanism of traditional Chinese medicine," Scientific Reports, vol. 6, no. 1, p. 21146, 2016.

[18] W. Tao, X. Xu, X. Wang et al., "Network pharmacology-based prediction of the active ingredients and potential targets of Chinese herbal_Radix Curcumae_formula for application to cardiovascular disease," Journal of Ethnopharmacology, vol. 145, no. 1, pp. 1-10, 2013.

[19] X. Xu, W. Zhang, C. Huang et al., "A novel chemometric method for the prediction of human oral bioavailability," International Journal of Molecular Sciences, vol. 13, no. 6, pp. 6964-6982, 2012.

[20] S. J. Yue, J. Liu, W. W. Feng et al., "System pharmacologybased dissection of the synergistic mechanism of Huangqi and Huanglian for diabetes mellitus," Frontiers in Pharmacology, vol. 8, p. 694, 2017.

[21] Y. Yao, X. Zhang, Z. Wang et al., "Deciphering the combination principles of Traditional Chinese Medicine from a systems pharmacology perspective based on Ma-huang Decoction," Journal of Ethnopharmacology, vol. 150, no. 2, pp. 619-638, 2013.

[22] X. X. Xu, J. P. Bi, L. Ping, P. Li, and F. Li, "A network pharmacology approach to determine the synergetic mechanisms of herb couple for treating rheumatic arthritis," Drug Design, Development and Therapy, vol. Volume 12, pp. 967-979, 2018.

[23] S. Pundir, M. J. Martin, C. O'Donovan, and The UniProt Consortium, "UniProt tools," Current Protocols in Bioinformatics, vol. 53, no. 1, pp. 1.29.1-1.29.15, 2016.

[24] S. Kim, J. Chen, T. Cheng et al., "PubChem 2019 update: improved access to chemical data," Nucleic Acids Research, vol. 47, no. D1, pp. D1102-D1109, 2019.

[25] C. Wang, Q. Ren, X. T. Chen et al., "System pharmacologybased strategy to decode the synergistic mechanism of Zhizhu Wan for functional dyspepsia," Frontiers in Pharmacology, vol. 9, p. 841, 2018.

[26] N. M. O'Boyle, M. Banck, C. A. James, C. Morley, T. Vandermeersch, and G. R. Hutchison, "Open Babel: an open chemical toolbox," Journal of Cheminformatics, vol. 3, no. 1, p. 33, 2011.

[27] A. Daina, O. Michielin, and V. Zoete, "SwissTargetPrediction: updated data and new features for efficient prediction of protein targets of small molecules," Nucleic Acids Research, vol. 47, no. W1, pp. W357-W364, 2019.
[28] K. G. Becker, K. C. Barnes, T. J. Bright, and S. A. Wang, "The genetic association database," Nature Genetics, vol. 36, no. 5, pp. 431-432, 2004.

[29] M. Shklar, L. Strichman-Almashanu, O. Shmueli, M. Shmoish, M. Safran, and D. Lancet, "GeneTide-Terra incognita discovery endeavor: a new transcriptome focused member of the GeneCards/GeneNote suite of databases," Nucleic Acids Research, vol. 33, no. Database issue, pp. D556-D561, 2005.

[30] M. Safran, I. Dalah, J. Alexander et al., "GeneCards version 3: the human gene integrator," Database, vol. 2010, p. baq020, 2010.

[31] J. Piñero, A. Bravo, N. Queralt-Rosinach et al., "DisGeNET: a comprehensive platform integrating information on human disease-associated genes and variants," Nucleic Acids Research, vol. 45, no. D1, pp. D833-D839, 2017.

[32] F. F. Cai, Y. Q. Bian, R. Wu et al., "Yinchenhao decoction suppresses rat liver fibrosis involved in an apoptosis regulation mechanism based on network pharmacology and transcriptomic analysis," Biomedicine \& Pharmacotherapy, vol. 114, p. 108863, 2019.

[33] S. H. Shi, Y. P. Cai, X. J. Cai et al., "A network pharmacology approach to understanding the mechanisms of action of traditional medicine: Bushenhuoxue formula for treatment of chronic kidney disease," PLoS One, vol. 9, no. 3, article e89123, 2014.

[34] G. Chen, C. Huang, Y. Liu et al., "A network pharmacology approach to uncover the potential mechanism of Yinchensini decoction," Evidence-based Complementary and Alternative Medicine, vol. 2018, 14 pages, 2018.

[35] D. Szklarczyk, A. L. Gable, D. Lyon et al., "STRING v11: protein-protein association networks with increased coverage, supporting functional discovery in genome-wide experimental datasets," Nucleic Acids Research, vol. 47, no. D1, pp. D607D613, 2019.

[36] G. Yu, L. G. Wang, Y. Han, and Q. Y. He, “clusterProfiler: an R package for comparing biological themes among gene clusters," Omics : a journal of integrative biology., vol. 16, no. 5, pp. 284-287, 2012.

[37] G. Cimmino, R. Tarallo, G. Nassa et al., "Activating stimuli induce platelet microRNA modulation and proteome reorganisation," Thrombosis and Haemostasis, vol. 114, no. 7, pp. 96-108, 2017.

[38] M. Sultan, S. Dökel, V. Amstislavskiy et al., "A simple strandspecific RNA-Seq library preparation protocol combining the Illumina TruSeq RNA and the dUTP methods," Biochemical and Biophysical Research Communications, vol. 422, no. 4, pp. 643-646, 2012.

[39] C. Xie, X. Mao, J. Huang et al., "KOBAS 2.0: a web server for annotation and identification of enriched pathways and diseases," Nucleic Acids Research, vol. 39, suppl_2, pp. W316W322, 2011.

[40] S. Li and B. Zhang, "Traditional Chinese medicine network pharmacology: theory, methodology and application," Chinese Journal of Natural Medicines, vol. 11, no. 2, pp. 110-120, 2013.

[41] B. Boezio, K. Audouze, P. Ducrot, and O. Taboureau, "Network-based approaches in pharmacology," Molecular Informatics, vol. 36, no. 10, 2017.

[42] X. Huang, G. C. Li, L. Yin, Z. H. Zhang, Y. X. Liang, and H. B. Chen, "The effective parts of liangxue tongyu prescription on cooling-blood and activating-blood and analysis of chemical constituents by HPLC-MS and GC-MS," Acta Pharmaceutica Sinica, vol. 50, no. 1, pp. 86-93, 2015. 
[43] R. Liu, J. A. Duan, W. U. Hao, P. Liu, E. X. Shang, and D. W. Qian, "Analysis and identification of water soluble components of water buffalo horn," Acta Pharmaceutica Sinica, vol. 50, no. 5, pp. 594-598, 2015.

[44] M. G. Liska, M. G. Crowley, J. P. Tuazon, and C. V. Borlongan, "Neuroprotective and neuroregenerative potential of pharmacologically-induced hypothermia with D-alanine Dleucine enkephalin in brain injury," Neural Regeneration Research, vol. 13, no. 12, pp. 2029-2037, 2018.

[45] Y. Zhang, B. Yi, J. Ma et al., "Quercetin promotes neuronal and behavioral recovery by suppressing inflammatory response and apoptosis in a rat model of intracerebral hemorrhage," Neurochemical Research, vol. 40, no. 1, pp. 195203, 2015.

[46] A. R. Galho, M. F. Cordeiro, S. A. Ribeiro et al., "Protective role of free and quercetin-loaded nanoemulsion against damage induced by intracerebral haemorrhage in rats," Nanotechnology, vol. 27, no. 17, p. 175101, 2016.

[47] C. López-Sánchez, F. J. Martín-Romero, F. Sun et al., "Blood micromolar concentrations of kaempferol afford protection against ischemia/reperfusion-induced damage in rat brain," Brain Research, vol. 1182, no. 1, pp. 123-137, 2007.

[48] L. Yu, C. Chen, L. F. Wang et al., "Neuroprotective effect of kaempferol glycosides against brain injury and neuroinflammation by inhibiting the activation of NF- $\kappa$ B and STAT3 in transient focal stroke," PLoS One, vol. 8, no. 2, article e55839, 2013.

[49] Q. Guo, S. Yang, D. Yang et al., "Differential mRNA expression combined with network pharmacology reveals network effects of Liangxue Tongyu Prescription for acute intracerebral hemorrhagic rats," Journal of Ethnopharmacology, vol. 246, p. $112231,2020$.

[50] J. A. G. Agúndez, M. Blanca, J. A. Cornejo-García, and E. García-Martín, "Pharmacogenomics of cyclooxygenases," Pharmacogenomics, vol. 16, no. 5, pp. 501-522, 2015.

[51] T. Grosser, S. Fries, and G. A. FitzGerald, "Biological basis for the cardiovascular consequences of COX-2 inhibition: therapeutic challenges and opportunities," The Journal of Clinical Investigation, vol. 116, no. 1, pp. 4-15, 2006.

[52] C. A. Worby and J. E. Dixon, "PTEN," Annual Review of Biochemistry, vol. 83, no. 1, pp. 641-669, 2014.

[53] W. Li, R. Huang, Z. Chen, L. J. Yan, J. W. Simpkins, and S. H. Yang, "PTEN degradation after ischemic stroke: a doubleedged sword," Neuroscience, vol. 274, pp. 153-161, 2014.

[54] K. Zhou, Q. Zhong, Y. C. Wang et al., "Regulatory T cells ameliorate intracerebral hemorrhage-induced inflammatory injury by modulating microglia/macrophage polarization through the IL-10/GSK3 $\beta /$ PTEN axis," Journal of Cerebral Blood Flow and Metabolism, vol. 37, no. 3, pp. 967-979, 2017.

[55] X. Wang, K. Chandrashekar, L. Wang et al., "Inhibition of nitric oxide synthase 1 induces salt-sensitive hypertension in nitric oxide synthase $1 \alpha$ knockout and wild-type mice," Hypertension, vol. 67, no. 4, pp. 792-799, 2016.

[56] N. Toda, K. Ayajiki, and T. Okamura, "Cerebral blood flow regulation by nitric oxide: recent advances," Pharmacological Reviews, vol. 61, no. 1, pp. 62-97, 2009.

[57] M. Willmot, C. Gibson, L. Gray, S. Murphy, and P. Bath, "Nitric oxide synthase inhibitors in experimental ischemic stroke and their effects on infarct size and cerebral blood flow: a systematic review," Free Radical Biology \& Medicine, vol. 39, no. 3, pp. 412-425, 2005.
[58] P. K. Liu, "Ischemia-reperfusion-related repair deficit after oxidative stress: implications of faulty transcripts in neuronal sensitivity after brain injury," Journal of Biomedical Science, vol. 10, no. 1, pp. 4-13, 2003.

[59] M. D'Amelio, V. Cavallucci, and F. Cecconi, "Neuronal caspase-3 signaling: not only cell death," Cell Death and Differentiation, vol. 17, no. 7, pp. 1104-1114, 2010.

[60] Z. Yanling, C. Kangning, S. Shuqin, and W. Ying, "Relationship between apoptosis and expression of caspase-3 after cerebral hemorrhage," Journal of the Fourth Military Medical University, vol. 25, no. 1, pp. 23-26, 2004.

[61] D. B. Sun, M. J. Xu, Q. M. Chen, and H. T. Hu, "Significant elevation of serum caspase-3 levels in patients with intracerebral hemorrhage," Clinica Chimica Acta, vol. 471, pp. 62-67, 2017.

[62] M. Chen, L. Lai, X. Li et al., "Baicalein attenuates neurological deficits and preserves blood-brain barrier integrity in a rat model of intracerebral hemorrhage," Neurochemical Research, vol. 41, no. 11, pp. 3095-3102, 2016.

[63] A. Bhinge, S. C. Namboori, X. Zhang, A. M. J. Vandongen, and L. W. Stanton, "Genetic correction of SOD1 mutant iPSCs reveals ERK and JNK activated AP1 as a driver of neurodegeneration in amyotrophic lateral sclerosis," Stem Cell Reports., vol. 8, no. 4, pp. 856-869, 2017.

[64] J. Sun and G. Nan, "The mitogen-activated protein kinase (MAPK) signaling pathway as a discovery target in stroke," Journal of Molecular Neuroscience, vol. 59, no. 1, pp. 90-98, 2016.

[65] Q. Jin, R. Li, N. Hu et al., "DUSP1 alleviates cardiac ische$\mathrm{mia} /$ reperfusion injury by suppressing the Mff- required mitochondrial fission and Bnip3-related mitophagy via the JNK pathways," Redox Biology, vol. 14, pp. 576-587, 2018.

[66] V. Atsaves, V. Leventaki, G. Z. Rassidakis, and F. X. Claret, "AP-1 transcription factors as regulators of immune responses in cancer," Cancers, vol. 11, no. 7, p. 1037, 2019.

[67] E. Shaulian and M. Karin, "AP-1 in cell proliferation and survival,” Oncogene, vol. 20, no. 19, pp. 2390-2400, 2001.

[68] X. Wei, W. Sun, R. Fan et al., "MEF2C regulates c-Jun but not TNF- $\alpha$ gene expression in stimulated mast cells," European Journal of Immunology, vol. 33, no. 10, pp. 2903-2909, 2003.

[69] G. Raivich, "C-Jun expression, activation and function in neural cell death, inflammation and repair," Journal of Neurochemistry, vol. 107, no. 4, pp. 898-906, 2008.

[70] J. Han, Y. Jiang, Z. Li, V. V. Kravchenko, and R. J. Ulevitch, "Activation of the transcription factor MEF2C by the MAP kinase p38 in inflammation," Nature, vol. 386, no. 6622, pp. 296-299, 1997.

[71] S. H. Yang, A. Galanis, and A. D. Sharrocks, “Targeting of p38 mitogen-activated protein kinases to MEF2 transcription factors," Molecular and Cellular Biology, vol. 19, no. 6, pp. 4028-4038, 1999.

[72] H. H. Wang, H. L. Hsieh, C. Y. Wu, C. C. Sun, and C. M. Yang, "Oxidized low-density lipoprotein induces matrix metalloproteinase-9 expression via a p42/p44 and JNKdependent AP-1 pathway in brain astrocytes," Glia, vol. 57, no. 1, pp. 24-38, 2009.

[73] M. Hammer, J. Mages, H. Dietrich et al., "Dual specificity phosphatase 1 (DUSP1) regulates a subset of LPS-induced genes and protects mice from lethal endotoxin shock," The Journal of Experimental Medicine, vol. 203, no. 1, pp. 15-20, 2006. 
[74] D. M. Taylor, R. Moser, E. Regulier et al., "MAP kinase phosphatase 1 (MKP-1/DUSP1) is neuroprotective in Huntington's disease via additive effects of JNK and p38 inhibition," The Journal of Neuroscience, vol. 33, no. 6, pp. 2313-2325, 2013.

[75] M. J. Berridge, “The inositol trisphosphate/calcium signaling pathway in health and disease," Physiological Reviews, vol. 96, no. 4, pp. 1261-1296, 2016.

[76] J. Liu, H. Yang, and B. Yu, "The correlation between blood calcium level, hematoma volume, stroke severity and prognosis in patients with acute cerebral hemorrhage," European Review for Medical and Pharmacological Sciences, vol. 20, no. 19, pp. 4119-4123, 2016.

[77] E. Sekerdag, I. Solaroglu, and Y. Gursoy-Ozdemir, "Cell death mechanisms in stroke and novel molecular and cellular treatment options," Current Neuropharmacology, vol. 16, no. 9, pp. 1396-1415, 2018.

[78] Y. Shi, "Mechanisms of caspase activation and inhibition during apoptosis," Molecular Cell, vol. 9, no. 3, pp. 459-470, 2002.

[79] M. K. McCoy and M. G. Tansey, "TNF signaling inhibition in the CNS: implications for normal brain function and neurodegenerative disease," Journal of Neuroinflammation, vol. 5, no. 1 , p. 45,2008 . 